\title{
A CONSTRUCTION OF A COMPLETE BOUNDED NULL CURVE IN $\mathrm{C}^{3}$
}

\author{
Leonor Ferrer, Francisco Martín, Masaaki Umehara and Kotaro Yamada
}

\begin{abstract}
We construct a complete bounded immersed null holomorphic curve in $\mathbf{C}^{3}$, which is a recovery of the previous paper of the last three authors on this subject.
\end{abstract}

\section{Introduction}

The study of global properties of complete complex null-curves is interesting from different points of view. Firstly, the real and imaginary part of such a curve are complete minimal surfaces in $\mathbf{R}^{3}$. Secondly, there exists a close relationship between null curves in $\mathbf{C}^{3}$ and surfaces of constant mean curvature $H=1$ in hyperbolic 3-space.

An important problem in the global theory of complete null curves is the so called Calabi-Yau problem, which deals with the existence of complete nullcurves inside a ball of $\mathbf{C}^{3}$. This problem was approached firstly in [5, Theorem A] using similar ideas to those used by Nadirashvili in [7] to solve the Calabi-Yau conjecture in $\mathbf{R}^{3}$. Unfortunately, the paper [5] has a mistake, and the first examples of complete bounded null curves in $\mathbf{C}^{3}$ were provided using other approach, by Alarcón and López [3]. Very recently, Alarcón and Forstnerič have got the most general results in this line (see $[1,2]$ ).

The purpose of this paper is to show that similar ideas to those given in [5] can be used to produce examples of complete bounded null holomorphic disks in a ball of $\mathbf{C}^{3}$ : In [5], Martín, Umehara and Yamada tried to construct a bounded holomorphic curve in $\operatorname{SL}(2, \mathbf{C})$ and used this example to get the desired bounded disk in $\mathbf{C}^{3}$. However, in this paper, we construct the bounded null curves directly in $\mathbf{C}^{3}$. In this aspect, our strategy is similar to that used by Alarcón and López in [3]. Although, as we mentioned before, these examples have been generalized in Alarcón and Forstnerič [2] by using different (and powerful)

2010 Mathematics Subject Classification. Primary 53A10, Secondary 53C42.

Key words and phrases. Bounded minimal surface, null curves.

The first and second authors are partially supported by MEC-FEDER Grant no. MTM201122547 and a Regional J. Andalucia Grant no. P09-FQM-5088. The third and the fourth authors are partially supported by Grant-in-Aid for Scientific Research (A) No. 22244006 and (B) No. 21340016, respectively, from Japan Society for the Promotion of Science.

Received December 18, 2012; revised May 14, 2013. 
methods, we think that the arguments and techniques exhibited in this paper are different from $[3,1,2]$, and might be of use in the solution of other questions related to the Calabi-Yau problem in different settings.

As applications of Theorem A in [5], the following objects were constructed;

(1) complete bounded minimal surfaces in the Euclidean 3 -space $\mathbf{R}^{3}$ ([5, Theorem A]),

(2) complete bounded holomorphic curves in $\mathbf{C}^{2}$ ([5, Corollary B $]$ ),

(3) weakly complete bounded maximal surfaces in the Lorentz-Minkowski 3-space $\mathbf{R}_{1}^{3}([5$, Corollary $\mathrm{D}])$,

(4) complete bounded null curves in $\operatorname{SL}(2, \mathbf{C})$ ([5, Theorem C $]$ ),

(5) complete bounded constant mean curvature one surfaces in the hyperbolic 3-space $H^{3}([5$, Theorem $\mathrm{C}])$.

We also constructed higher genus examples of the first three objects in [6]. All of these applications in [5] and [6] are correct as a consequence.

\section{The Main Theorem and the Key Lemma}

We denote by $($,$) (resp. \langle\rangle$,$) the C-bilinear inner product (resp. the$ Hermitian inner product) of $\mathbf{C}^{3}$ :

$$
(\boldsymbol{x}, \boldsymbol{y}):=x_{1} y_{1}+x_{2} y_{2}+x_{3} y_{3}, \quad\langle\boldsymbol{x}, \boldsymbol{y}\rangle:=(\boldsymbol{x}, \overline{\boldsymbol{y}}),
$$

where $\boldsymbol{x}=\left(x_{1}, x_{2}, x_{3}\right), \boldsymbol{y}=\left(y_{1}, y_{2}, y_{3}\right) \in \mathbf{C}^{3}$, and $\overline{\boldsymbol{y}}$ denotes the complex conjugate of $y$.

Remark 1.1. In this paper, we identify an element of $\mathbf{C}^{3}$ with a column vector when the matrix product is used.

The Hermitian norm of $\mathbf{C}^{3}$ is denoted by $|\boldsymbol{x}|:=\sqrt{\langle\boldsymbol{x}, \boldsymbol{x}\rangle}$ for $\boldsymbol{x} \in \mathbf{C}^{3}$. In particular, it holds that

$$
|(\boldsymbol{x}, \boldsymbol{y})|=|\langle\boldsymbol{x}, \overline{\boldsymbol{y}}\rangle| \leq|\boldsymbol{x}||\overline{\boldsymbol{y}}|=|\boldsymbol{x}||\boldsymbol{y}| .
$$

Let $\mathbf{M}(3, \mathbf{C})$ (resp. $\mathbf{M}(3, \mathbf{R}))$ be the set of complex (resp. real) $(3 \times 3)$-matrices. Moreover, we will use the following notation for the set of complex (resp. special) orthogonal matrices

$$
\begin{aligned}
& \mathrm{O}(3, \mathbf{C}):=\left\{A \in \mathrm{M}(3, \mathbf{C}) ; A^{t} A=\mathrm{id}\right\} \\
& \text { (resp. } \left.\mathrm{SO}(3):=\left\{A \in \mathrm{M}(3, \mathbf{R}) ; A^{t} A=\mathrm{id}, \operatorname{det} A=1\right\}\right),
\end{aligned}
$$

where $A^{t}$ means the transposed matrix of $A$. As usual, we denote $\mathrm{U}(3):=$ $\left\{A \in \mathrm{M}(3, \mathbf{C}) ; A^{*} A=\mathrm{id}\right\}$, where $A^{*}$ is the conjugate transposed matrix of $A$. For each $A \in \mathrm{M}(3, \mathbf{C})$, we define the matrix norm as

$$
\|A\|:=\sup _{\boldsymbol{x} \in \mathbf{C}^{3} \backslash\{\boldsymbol{0}\}} \frac{|A \boldsymbol{x}|}{|\boldsymbol{x}|} .
$$


If $A \in \mathrm{M}(3, \mathbf{C})$ is a non-singular matrix,

$$
\frac{1}{\left\|A^{-1}\right\|}|\boldsymbol{x}| \leq|A \boldsymbol{x}| \leq\|A\||\boldsymbol{x}|
$$

holds. It is well-known that

$$
\|A\|=\sqrt{\max \left\{\mu_{1}, \mu_{2}, \mu_{3}\right\}} \quad(A \in \mathbf{M}(3, \mathbf{C}))
$$

holds, where $\mu_{j} \in \mathbf{R}(j=1,2,3)$ are the eigenvalues of the positive semi-definite Hermitian matrix $A^{*} A$.

A holomorphic map $F: D \rightarrow \mathbf{C}^{3}$, defined on a domain $D \subset \mathbf{C}$, is a null immersion if and only if

(1.6) $\left(\varphi_{F}, \varphi_{F}\right)=0 \quad$ and $\left|\varphi_{F}\right|^{2}=\left\langle\varphi_{F}, \varphi_{F}\right\rangle>0, \quad$ where $\varphi=\varphi_{F}:=\frac{d F}{d z}$,

and $z$ is the canonical complex coordinate of $\mathbf{C}$. In this case the pull-back of the Hermitian metric of $\mathbf{C}^{3}$ by $F$ is expressed as

$$
d s_{F}^{2}:=\langle d F, d F\rangle=\left|\varphi_{F}\right|^{2}|d z|^{2},
$$

which is called the induced metric of $F$. For a holomorphic null immersion $F: D \rightarrow \mathbf{C}^{3}$, the first equality of (1.6) implies that there exist a meromorphic function $g$ and a holomorphic function $\eta$ such that

$$
\varphi_{F}=\frac{1}{2}\left(1-g^{2}, \mathrm{i}\left(1+g^{2}\right), 2 g\right) \eta \quad(\mathrm{i}=\sqrt{-1}) .
$$

We call $(g, \eta)$ the Weierstrass data of $F$. Using these data, the induced metric (1.7) is expressed as

$$
d s_{F}^{2}=\frac{1}{2}\left(1+|g|^{2}\right)^{2}|\eta|^{2}|d z|^{2} .
$$

Throughout this paper, we denote the open (resp. closed) disc on $\mathbf{C}$ centered at 0 with radius $r$ by

$$
\mathbf{D}_{r}:=\{z \in \mathbf{C} ;|z|<r\}, \quad\left(\text { resp. } \overline{\mathbf{D}}_{r}:=\{z \in \mathbf{C} ;|z| \leq r\}\right) \quad(r>0) .
$$

The goal of this paper is to prove the following

THEOREM 1.2 (The Main Theorem). There exists a holomorphic null immersion $f: \mathbf{D}_{1} \rightarrow \mathbf{C}^{3}$ such that the induced metric $d s_{f}^{2}$ is complete, and the image $f\left(\mathbf{D}_{1}\right)$ is bounded in $\mathbf{C}^{3}$.

Theorem A in [5] is the same statement as our main Theorem 1.2, and the purpose of this paper is to give a correct proof of it as a recovery of the previous proof given in [5]. As in [5, (3.1)], the transformation

$$
\mathscr{T}:\left\{\left(x_{1}, x_{2}, x_{3}\right) \in \mathbf{C}^{3} ; x_{3} \neq 0\right\} \rightarrow\left\{\left(y_{i j}\right) \in \operatorname{SL}(2, \mathbf{C}) ; y_{11} \neq 0\right\}
$$


defined by

$$
\mathscr{T}\left(x_{1}, x_{2}, x_{3}\right):=\frac{1}{x_{3}}\left(\begin{array}{cc}
1 & x_{1}+\mathrm{i} x_{2} \\
x_{1}-\mathrm{i} x_{2} & \left(x_{1}\right)^{2}+\left(x_{2}\right)^{2}+\left(x_{3}\right)^{2}
\end{array}\right)
$$

maps null curves in $\mathbf{C}^{3}$ to null curves in $\operatorname{SL}(2, \mathbf{C})$, where a holomorphic map $F: \mathbf{D}_{1} \rightarrow \mathrm{SL}(2, \mathbf{C})$ is called null if the determinant of the matrix $d F / d z$ vanishes. By Theorem 1.2, there exists a complete bounded null immersion $f: \mathbf{D}_{1} \rightarrow \mathbf{C}^{3}$. We may assume that the image of $f$ lies in $\left\{\left(x_{1}, x_{2}, x_{3}\right) \in \mathbf{C}^{3} ; x_{3} \neq 0\right\}$ by a suitable translation. Then $\mathscr{T} \circ f$ gives a bounded null immersed curve in $\mathrm{SL}(2, \mathbf{C})$. Moreover, the pull-back metric of the canonical Hermitian metric on $\operatorname{SL}(2, \mathbf{C})$ by $\mathscr{T} \circ f$ is complete by [5, Lemma 3.1], which proves the assertion (4) in the introduction. Applying the well-known Bryant representation formula (cf. [5, Page 123]), the projection of $\mathscr{T} \circ f$ into the hyperbolic 3-space $H^{3}=\mathrm{SL}(2, \mathrm{C}) / \mathrm{SU}(2)$ gives a complete bounded constant mean curvature one immersed disc in $H^{3}$, which proves the assertion (5) in the introduction. The proofs given in [5, 6] of Assertions (1), (2), (3) in the introduction, as applications of Theorem 1.2, are all correct without any modifications.

Theorem 1.2 can be proved by the following proposition in the same way as [7, 4]. So, an iterative application of Proposition 1.3 for an initial immersion $f_{0}: \mathbf{D}_{1} \rightarrow \mathbf{C}^{3}$, provides us a sequence of bounded null discs whose intrinsic diameter goes to infinity. The desired immersion $f$ is obtained as a limit of such a sequence. The initial immersion of such an iteration can be chosen arbitrarily. In fact, one can choose $f_{0}: \mathbf{D}_{1} \ni z \mapsto(z, \mathbf{i} z, 0) \in \mathbf{C}^{3}$. If we choose another initial immersion, one can expect a different complete null immersion.

Proposition 1.3. Let $X: \overline{\mathbf{D}}_{1} \rightarrow \mathbf{C}^{3}$ be a holomorphic null immersion of the closed disc $\overline{\mathbf{D}}_{1} \subset \mathbf{C}$ into $\mathbf{C}^{3}$. Suppose that there exist positive numbers $\rho$ and $r$ such that

$(\mathrm{X}-1) \quad X(0)=0$,

(X-2) $\left(\mathbf{D}_{1}, d s_{X}^{2}\right)$ contains the geodesic disc centered at 0 and of radius $\rho$,

(X-3) and $|X| \leq r$ holds on $\overline{\mathbf{D}}_{1}$.

Then, for an arbitrary given positive numbers $\varepsilon$ and $s$, there exists a holomorphic null immersion $Y: \overline{\mathbf{D}}_{1} \rightarrow \mathbf{C}^{3}$ satisfying

(Y-1) $\left|\varphi_{Y}-\varphi_{X}\right|<\varepsilon$ and $|Y-X|<\varepsilon$ hold on $\mathbf{D}_{1-\varepsilon}$, where $\varphi_{X}=d X / d z$ and $\varphi_{Y}=d Y / d z$

$(\mathrm{Y}-2)\left(\mathbf{D}_{1}, d s_{Y}^{2}\right)$ contains the geodesic disc $\mathscr{D}$ centered at 0 with radius $\rho+s$,

$(\mathrm{Y}-3)$ and on the boundary $\partial \mathscr{D}$ of the geodesic disc $\mathscr{D}$ in $(\mathrm{Y}-2)$, it holds that $|Y| \leq \sqrt{r^{2}+s^{2}}+\varepsilon$.

This proposition is a consequence of the following Key Lemma. (The proof of Proposition 1.3 is given in Section 4.) To explain it, we will define three constants

$$
N=N(\rho, r, \mu, v, s, \varepsilon), \quad C_{1}=C_{1}(v) \quad \text { and } \quad C_{2}=C_{2}(\mu)
$$


depending on six positive constants $\rho, r, \mu, v, s, \varepsilon$ as follows (cf. (1.11), (1.13)$(1.15))$. Here $\rho$ and $r$ have been already given in (X-2) and (X-3), and we will fix $\mu, v$ in the statement of Lemma 1.4. The remaining two constants $s, \varepsilon$ are arbitrary in the statement of Lemma 1.4, but will coincide with the corresponding constants as in Proposition 1.3.

The constants $C_{1}$ and $C_{2}$ are set as

$$
C_{1}:=\frac{v}{5}, \quad C_{2}:=6\left(\mu^{2}+2 \mu+2\right) .
$$

Next, we set

$$
\begin{aligned}
& c_{1}:=6 \mu^{2}+12 \mu+8, \quad c_{2}:=3 \mu+\frac{2 \varepsilon(\rho+s)}{C_{1}}, \\
& c_{3}:=\frac{s \alpha+\frac{\alpha^{2}}{2}+2 r \varepsilon+2 \varepsilon^{2}}{\sqrt{r^{2}+s^{2}}} \quad\left(\alpha:=c_{2}+5 \varepsilon+(r+2 \varepsilon) \sqrt{2 C_{2}}\right) .
\end{aligned}
$$

We then choose an integer $N$ so that it satisfies the following three inequalities;

$$
\begin{aligned}
& N \geq \max \left\{36, \frac{2 \varepsilon}{v}, \varepsilon,(12 \mu)^{2},\left[\frac{2^{5}(3 \mu+\varepsilon)}{v}\left(2+\frac{6 \mu+2 \varepsilon}{3 v}\right)\right]^{4}\right\}, \\
& N \geq \max \left\{\frac{3}{\varepsilon},\left(\frac{2 \varepsilon}{C_{1}}\right)^{4},\left(\frac{1}{v}\left(\varepsilon+\frac{C_{1}}{2}\right)\right)^{4 / 3},\left(\frac{2(\rho+s)}{C_{1}}\right)^{4}\right\} \\
& N \geq \max \left\{\left(\frac{c_{3}+2 \varepsilon}{\varepsilon}\right)^{4},\left(\frac{1+c_{2}+6 \mu+3 \varepsilon}{\varepsilon}\right)^{4}\right\}
\end{aligned}
$$

Lemma 1.4 (The Key Lemma). Assume a holomorphic null immersion $X: \overline{\mathbf{D}}_{1} \rightarrow \mathbf{C}^{3}$ and positive real numbers $\rho$ and $r$ satisfy $(\mathrm{X}-1)-(\mathrm{X}-3)$. We set

$$
v:=\min _{\overline{\mathbf{D}}_{1}}\left|\varphi_{X}\right|>0, \quad \mu:=\max \left\{1+\max _{\overline{\mathbf{D}}_{1}}\left|\varphi_{X}\right|, \max _{\overline{\mathbf{D}}_{1}}\left|\varphi_{X}^{\prime}\right|\right\},
$$

where $\varphi_{X}:=X^{\prime}=d X / d z$ and $\varphi_{X}^{\prime}:=d \varphi_{X} / d z$. For arbitrary positive numbers $\varepsilon$ and $s$, we take positive constants $C_{1}, C_{2}$ and a positive integer $N$ as in (1.11), (1.13)-(1.15). Then there exist a sequence $\left\{F_{j}\right\}_{j=0, \ldots, 2 N}$ of holomorphic null immersions $F_{j}: \overline{\mathbf{D}}_{1} \rightarrow \mathbf{C}^{3}$ and a sequence $\left\{\boldsymbol{v}_{j}\right\}_{j=1, \ldots, 2 N}$ of unit vectors in $\mathbf{C}^{3}$ which satisfy the following assertions $(\mathrm{K}-0)-(\mathrm{K}-6)$, where the compact set $\omega_{j} \subset \mathbf{C}$, an open neighborhood $\varpi_{j}$ of $\omega_{j}$ and the "base point" $\zeta_{j}$ of $\varpi_{j}$ are as in (A.8) and (A.9) in Appendix A, and

$$
\varphi_{l}=\frac{d F_{l}}{d z} \quad(l=0, \ldots, 2 N)
$$

$(\mathrm{K}-0) F_{0}=X$.

$(\mathrm{K}-1) F_{l}(0)=0(l=0, \ldots, 2 N)$. 
$(\mathrm{K}-2)\left|\varphi_{l}-\varphi_{l-1}\right| \leq \frac{\varepsilon}{2 N^{2}}$ holds on $\overline{\mathbf{D}}_{1} \backslash \varpi_{l}$ for each $l=1, \ldots, 2 N$.

(K-3) The inequality

$$
\left|\varphi_{l}\right| \geq \begin{cases}C_{1} N^{9 / 4} & \text { on } \omega_{l}, \\ C_{1} N^{-3 / 4} & \text { on } \bar{\varpi}_{l}\end{cases}
$$

holds for each $l=1, \ldots, 2 N$, where $\bar{\varpi}_{l}$ is the closure of the $\varpi_{l}$, see Appendix $A$.

(K-4) $\left|\left(\boldsymbol{v}_{l}, \boldsymbol{v}_{l}\right)\right| \geq 1 / N^{1 / 4}$ holds for each $l=1, \ldots, 2 N$.

(K-5) $\left|F_{l-1}\left(\zeta_{l}\right)\right|<1 / \sqrt{N}$, or

$$
\left|\left\langle\frac{F_{l-1}(p)}{\left|F_{l-1}(p)\right|}, \boldsymbol{v}_{l}\right\rangle\right| \geq 1-\frac{C_{2}}{\sqrt{N}} \quad\left(\text { on } \bar{\varpi}_{l}\right)
$$

holds for each $l=1, \ldots, 2 N$.

$(\mathrm{K}-6)\left\langle F_{l-1}, \boldsymbol{v}_{l}\right\rangle=\left\langle F_{l}, \boldsymbol{v}_{l}\right\rangle$ holds on $\overline{\mathbf{D}}_{1}$ for each $l=1, \ldots, 2 N$.

In the proof of the Key Lemma 1.4, we use the notion of Gauss maps of holomorphic null immersions: Let $F: D \rightarrow \mathbf{C}^{3}$ be a holomorphic null immersion of a domain $D \subset \mathbf{C}$. Then both the real part $\operatorname{Re} F$ and the imaginary part $\operatorname{Im} F$ give conformal minimal immersions into $\mathbf{R}^{3}$ with the same Gauss map. So we call the Gauss map $G: D \rightarrow S^{2}$ of both $\operatorname{Re} F$ and $\operatorname{Im} F$ the Gauss map of $F$, where $S^{2} \subset \mathbf{R}^{3}$ is the unit sphere. Then $G$ is expressed as

$$
G=\frac{-\mathrm{i}(\varphi \times \bar{\varphi})}{|\varphi|^{2}}: D \rightarrow S^{2} \subset \mathbf{R}^{3} \quad\left(\varphi=\frac{d F}{d z}\right),
$$

because (1.6) implies that $|\varphi \times \bar{\varphi}|=|\varphi|^{2}$, where " $\times$ " denotes the complexification of the vector product of $\mathbf{R}^{3}$. Using the Weierstrass data (1.8), $G$ is expressed as

$$
G=\left(\frac{2 \operatorname{Re} g}{1+|g|^{2}}, \frac{2 \operatorname{Im} g}{1+|g|^{2}}, \frac{|g|^{2}-1}{1+|g|^{2}}\right) .
$$

That is, $g=\pi_{S} \circ G$, where $\pi_{S}: S^{2} \rightarrow \mathbf{C} \cup\{\infty\}$ is the stereographic projection from the north pole.

\section{Preliminary estimates}

Let $F_{0}=X: \overline{\mathbf{D}}_{1} \rightarrow \mathbf{C}^{3}$ be a holomorphic null immersion as in the assumption of the Key Lemma 1.4. Here, we prepare some basic properties of $\left\{F_{j}\right\}_{j=0, \ldots, 2 N}$ in the conclusion of the Key Lemma 1.4.

LEMma 2.1. If (K-1) and (K-2) in the Key Lemma 1.4 are satisfied for $l \in\{1, \ldots, 2 N\}$, then

$$
\left|F_{l}-F_{l-1}\right| \leq \frac{\varepsilon}{N^{2}} \quad \text { on } \overline{\mathbf{D}}_{1} \backslash \varpi_{l}
$$


Proof. Let $p \in \overline{\mathbf{D}}_{1} \backslash \varpi_{l}$. Then there exists a path $\gamma$ in $\overline{\mathbf{D}}_{1} \backslash \varpi_{l}$ joining 0 and $p$ whose Euclidean length is not greater than $1+\frac{\pi}{N}$ (see Lemma A.2 in Appendix
A). Thus, we have

$$
\begin{array}{rlrl}
\left|F_{l}(p)-F_{l-1}(p)\right| & =\left|\int_{\gamma}\left(\varphi_{l}(z)-\varphi_{l-1}(z)\right) d z\right| & \quad \text { by }(\mathrm{K}-1)) \\
& \leq \int_{\gamma}\left|\varphi_{l}(z)-\varphi_{l-1}(z)\right||d z| \leq \operatorname{Length}_{\mathbf{C}}(\gamma) \frac{\varepsilon}{2 N^{2}} & (\text { by }(\mathrm{K}-2)) \\
& \left.\leq\left(1+\frac{\pi}{N}\right) \frac{\varepsilon}{2 N^{2}} \leq 2 \cdot \frac{\varepsilon}{2 N^{2}}=\frac{\varepsilon}{N^{2}} \quad \text { (by }(1.13)\right)
\end{array}
$$

where $\operatorname{Length}_{\mathbf{C}}(\gamma)$ is the length of $\gamma$ with respect to the metric $|d z|^{2}$ on $\mathbf{C}$.

Lemma 2.2. Fix an integer $j(1 \leq j \leq 2 N)$. If $F_{0}, F_{1}, \ldots F_{j-1}$ satisfy $(\mathrm{K}-0)$ and $(\mathrm{K}-2)$ of the Key Lemma 1.4. Then

$$
\left|\varphi_{j-1}\right| \geq \frac{v}{2} \quad \text { and } \quad\left|\varphi_{j-1}\right| \leq \mu \quad\left(\text { on } \quad \overline{\mathbf{D}}_{1} \backslash\left(\varpi_{1} \cup \cdots \cup \varpi_{j-1}\right)\right)
$$

hold, where $\mu$ and $v$ are constants defined in (1.16).

Proof. By (K-0), (K-2), (1.16) and (1.13),

$$
\begin{aligned}
\left|\varphi_{j-1}\right| & \geq\left|\varphi_{0}\right|-\left|\varphi_{1}-\varphi_{0}\right|-\cdots-\left|\varphi_{j-1}-\varphi_{j-2}\right| \\
& \geq \min _{\overline{\mathbf{D}}_{1}}\left|\varphi_{0}\right|-\frac{(j-1) \varepsilon}{2 N^{2}} \geq v-\frac{\varepsilon}{N} \geq \frac{v}{2}
\end{aligned}
$$

holds on $\overline{\mathbf{D}}_{1} \backslash\left(\varpi_{1} \cup \cdots \cup \varpi_{j-1}\right)$. On the other hand, we have

$$
\begin{aligned}
\left|\varphi_{j-1}\right| & \leq\left|\varphi_{0}\right|+\left|\varphi_{1}-\varphi_{0}\right|+\cdots+\left|\varphi_{j-1}-\varphi_{j-2}\right| \\
& \leq \max _{\overline{\mathbf{D}}_{1}}\left|\varphi_{0}\right|+\frac{(j-1) \varepsilon}{2 N^{2}} \leq \max _{\overline{\mathbf{D}}_{1}}\left|\varphi_{0}\right|+\frac{\varepsilon}{N} \leq \max _{\overline{\mathbf{D}}_{1}}\left|\varphi_{0}\right|+1 \leq \mu .
\end{aligned}
$$

LEMMA 2.3. Fix an integer $j(1 \leq j \leq 2 N)$. If $F_{0}, F_{1}, \ldots F_{j-1}$ satisfy (K-0) and (K-2) of the Key Lemma 1.4. Then for each $p \in \bar{\varpi}_{j}$, it holds that

$$
\left|F_{j-1}(p)-F_{j-1}\left(\zeta_{j}\right)\right| \leq \frac{6 \mu}{N}, \quad\left|\varphi_{j-1}(p)-\varphi_{j-1}\left(\zeta_{j}\right)\right| \leq \frac{6 \mu+2 \varepsilon}{N},
$$

where $\zeta_{j}$ is the "base point" of $\varpi_{j}$ given in (A.9) of Appendix A. 
Proof. By Lemma A.3 in Appendix A, there exists a path $\gamma$ in $\bar{\varpi}_{j}$ joining $\zeta_{j}$ and $p$ such that $\operatorname{Length}_{\mathbf{C}}(\gamma) \leq 6 / N$. Since the image of $\gamma$ lies on $\overline{\mathbf{D}}_{1} \backslash\left(\varpi_{1} \cup \cdots \cup \varpi_{j-1}\right)$, Lemma 2.2 implies that

$$
\left|F_{j-1}(p)-F_{j-1}\left(\zeta_{j}\right)\right| \leq \int_{\gamma}\left|\varphi_{j-1}(z)\right||d z| \leq \mu \cdot \operatorname{Length}_{\mathbf{C}}(\gamma) \leq \frac{6 \mu}{N} .
$$

On the other hand,

$$
\begin{aligned}
\left|\varphi_{j-1}(p)-\varphi_{j-1}\left(\zeta_{j}\right)\right| & \\
\leq & \left|\varphi_{j-1}(p)-\varphi_{j-2}(p)\right|+\cdots+\left|\varphi_{1}(p)-\varphi_{0}(p)\right|+\left|\varphi_{0}(p)-\varphi_{0}\left(\zeta_{j}\right)\right| \\
& +\left|\varphi_{j-1}\left(\zeta_{j}\right)-\varphi_{j-2}\left(\zeta_{j}\right)\right|+\cdots+\left|\varphi_{1}\left(\zeta_{j}\right)-\varphi_{0}\left(\zeta_{j}\right)\right| \\
\leq & \frac{2(j-1) \varepsilon}{2 N^{2}}+\left|\varphi_{0}(p)-\varphi_{0}\left(\zeta_{j}\right)\right| \leq \frac{2 \varepsilon}{N}+\left|\int_{\gamma} \varphi_{0}^{\prime}(z) d z\right| \quad(\text { by }(\mathrm{K}-2)) \\
\leq & \frac{2 \varepsilon}{N}+\int_{\gamma}\left|\varphi_{0}^{\prime}(z)\right||d z| \leq \frac{2 \varepsilon}{N}+\mu \operatorname{Length}_{\mathbf{C}}(\gamma) \leq \frac{2 \varepsilon}{N}+\frac{6 \mu}{N} \quad(\text { by }(1.16)) .
\end{aligned}
$$

We fix $j(1 \leq j \leq 2 N)$ and assume $X=F_{0}, F_{1}, \ldots F_{j-1}$ are already constructed and satisfy $(\mathrm{K}-0)-(\mathrm{K}-6)$. From now on, we give a recipe of construction of $F_{j}$ and $\boldsymbol{v}_{j}$ as an inductive procedure:

LEMMA 2.4. There exists a unit vector $\boldsymbol{u} \in \mathbf{C}^{3}$ (i.e. $|\boldsymbol{u}|=1$ ) such that

(1) $\delta^{2}:=|(\boldsymbol{u}, \boldsymbol{u})| \geq 1 / N^{1 / 4}$.

(2) If

$$
\left|F_{j-1}\left(\zeta_{j}\right)\right| \geq \frac{1}{\sqrt{N}}
$$

it holds that

$$
\left|\left\langle\frac{F_{j-1}(p)}{\left|F_{j-1}(p)\right|}, \boldsymbol{u}\right\rangle\right| \geq 1-\frac{c_{1}}{\sqrt{N}} \quad\left(p \in \bar{\varpi}_{j}\right),
$$

where $c_{1}$ is the constant given in (1.12).

Proof. When $\left|F_{j-1}\left(\zeta_{j}\right)\right|<1 / \sqrt{N}$, the unit vector $\boldsymbol{u}=(0,0,1)$ satisfies the conclusions. (Note that the conclusion (2) is empty in this case.)

Now, we assume (2.1), and set

$$
\boldsymbol{u}_{0}:=\frac{F_{j-1}\left(\zeta_{j}\right)}{\left|F_{j-1}\left(\zeta_{j}\right)\right|}
$$

By Lemma 2.3,

$$
\left|F_{j-1}(p)-F_{j-1}\left(\zeta_{j}\right)\right| \leq \frac{6 \mu}{N}
$$

holds for each $p \in \bar{\varpi}_{j}$. Then, for $p \in \bar{\varpi}_{j}$, it holds that 


$$
\begin{aligned}
\left|F_{j-1}(p)\right| & \geq\left|F_{j-1}\left(\zeta_{j}\right)\right|-\left|F_{j-1}(p)-F_{j-1}\left(\zeta_{j}\right)\right| \geq\left|F_{j-1}\left(\zeta_{j}\right)\right|-\frac{6 \mu}{N} \\
& \geq\left|F_{j-1}\left(\zeta_{j}\right)\right|\left(1-\frac{6 \mu}{N\left|F_{j-1}\left(\zeta_{j}\right)\right|}\right) \geq\left|F_{j-1}\left(\zeta_{j}\right)\right|\left(1-\frac{6 \mu}{N \frac{1}{\sqrt{N}}}\right) \\
& =\left|F_{j-1}\left(\zeta_{j}\right)\right|\left(1-\frac{6 \mu}{\sqrt{N}}\right) \geq \frac{1}{2}\left|F_{j-1}\left(\zeta_{j}\right)\right|
\end{aligned}
$$

Thus, using (2.1) again, we have

$$
\left|F_{j-1}(p)\right| \geq \frac{1}{2}\left|F_{j-1}\left(\zeta_{j}\right)\right| \geq \frac{1}{2 \sqrt{N}} \quad\left(p \in \bar{\varpi}_{j}\right) .
$$

Then by the relationship of the arithmetic mean and the geometric mean, we have

$$
\begin{aligned}
\frac{(6 \mu)^{2}}{N^{2}} & \left.\geq\left|F_{j-1}(p)-F_{j-1}\left(\zeta_{j}\right)\right|^{2} \quad \text { (by }(2.3)\right) \\
& =\left|F_{j-1}(p)\right|^{2}+\left|F_{j-1}\left(\zeta_{j}\right)\right|^{2}-2 \operatorname{Re}\left\langle F_{j-1}(p), F_{j-1}\left(\zeta_{j}\right)\right\rangle \\
& =\left|F_{j-1}(p)\right|\left|F_{j-1}\left(\zeta_{j}\right)\right|\left(\frac{\left|F_{j-1}(p)\right|}{\left|F_{j-1}\left(\zeta_{j}\right)\right|}+\frac{\left|F_{j-1}\left(\zeta_{j}\right)\right|}{\left|F_{j-1}(p)\right|}-2 \operatorname{Re}\left\langle\frac{F_{j-1}(p)}{\left|F_{j-1}(p)\right|}, \frac{F_{j-1}\left(\zeta_{j}\right)}{\left|F_{j-1}\left(\zeta_{j}\right)\right|}\right\rangle\right) \\
& \geq 2\left|F_{j-1}(p)\right|\left|F_{j-1}\left(\zeta_{j}\right)\right|\left(1-\operatorname{Re}\left\langle\frac{F_{j-1}(p)}{\left|F_{j-1}(p)\right|}, \frac{F_{j-1}\left(\zeta_{j}\right)}{\left|F_{j-1}\left(\zeta_{j}\right)\right|}\right\rangle\right) \\
& \geq \frac{1}{N}\left(1-\operatorname{Re}\left\langle\frac{F_{j-1}(p)}{\left|F_{j-1}(p)\right|}, \frac{F_{j-1}\left(\zeta_{j}\right)}{\left|F_{j-1}\left(\zeta_{j}\right)\right|}\right\rangle\right) \quad(\text { by }(2.4),(2.1)) \\
& \geq \frac{1}{N}\left(1-\left|\left\langle\frac{F_{j-1}(p)}{\left|F_{j-1}(p)\right|}, \frac{F_{j-1}\left(\zeta_{j}\right)}{\left|F_{j-1}\left(\zeta_{j}\right)\right|}\right\rangle\right|\right) .
\end{aligned}
$$

Hence, by (1.13), we have

$$
\begin{aligned}
\left|\left\langle\frac{F_{j-1}(p)}{\left|F_{j-1}(p)\right|}, \boldsymbol{u}_{0}\right\rangle\right| & =\left|\left\langle\frac{F_{j-1}(p)}{\left|F_{j-1}(p)\right|}, \frac{F_{j-1}\left(\zeta_{j}\right)}{\left|F_{j-1}\left(\zeta_{j}\right)\right|}\right\rangle\right| \\
& \geq 1-\frac{(6 \mu)^{2}}{N}=1-\frac{6 \mu^{2}}{\sqrt{N}} \frac{6}{\sqrt{N}} \geq 1-\frac{6 \mu^{2}}{\sqrt{N}} .
\end{aligned}
$$

CASE A. We consider the case $\left|\left(\boldsymbol{u}_{0}, \boldsymbol{u}_{0}\right)\right| \geq 1 / N^{1 / 4}$. In this case, we set $\boldsymbol{u}=\boldsymbol{u}_{0} . \quad$ Then the unit vector $\boldsymbol{u}$ satisfies (1) trivially. Moreover, (2.5) implies the assertion (2) because $c_{1}$ in (1.12) satisfies $c_{1} \geq 6 \mu^{2}$.

CASE B. We next consider the case $\left|\left(\boldsymbol{u}_{0}, \boldsymbol{u}_{0}\right)\right|<1 / N^{1 / 4}$. In this case, set

$$
\boldsymbol{u}:=\frac{\tilde{\boldsymbol{u}}}{|\tilde{\boldsymbol{u}}|}, \quad \text { where } \tilde{\boldsymbol{u}}:=\boldsymbol{u}_{0}+\frac{2}{N^{1 / 4}} \overline{\boldsymbol{u}_{0}} .
$$


To show (1) and (2), we set

$$
\delta_{0}^{2}:=\left|\left(\boldsymbol{u}_{0}, \boldsymbol{u}_{0}\right)\right|\left(<\frac{1}{N^{1 / 4}}\right) .
$$

Since $\boldsymbol{u}_{0}$ is a unit vector, (2.6) yields

$$
\begin{aligned}
|(\tilde{\boldsymbol{u}}, \tilde{\boldsymbol{u}})| & =\left|\left(\boldsymbol{u}_{0}, \boldsymbol{u}_{0}\right)+\frac{4}{\sqrt{N}}\left(\overline{\boldsymbol{u}_{0}}, \overline{\boldsymbol{u}_{0}}\right)+\frac{4}{N^{1 / 4}}\left(\boldsymbol{u}_{0}, \overline{\boldsymbol{u}_{0}}\right)\right| \\
& \geq \frac{4}{N^{1 / 4}}\left|\left\langle\boldsymbol{u}_{0}, \boldsymbol{u}_{0}\right\rangle\right|-\left|\left(\boldsymbol{u}_{0}, \boldsymbol{u}_{0}\right)\right|-\frac{4}{\sqrt{N}}\left|\left(\boldsymbol{u}_{0}, \boldsymbol{u}_{0}\right)\right| \\
& =\frac{4}{N^{1 / 4}}-\delta_{0}^{2}\left(1+\frac{4}{\sqrt{N}}\right) \geq \frac{4}{N^{1 / 4}}-\frac{5}{3} \delta_{0}^{2} \geq \frac{7}{3 N^{1 / 4}}
\end{aligned}
$$

On the other hand, using (2.7) and (1.13) again, we have

$$
\begin{aligned}
|\tilde{\boldsymbol{u}}|^{2} & =\left|\boldsymbol{u}_{0}\right|^{2}+\frac{4}{\sqrt{N}}\left|\overline{\boldsymbol{u}_{0}}\right|^{2}+\frac{4}{N^{1 / 4}} \operatorname{Re}\left(\left\langle\boldsymbol{u}_{0}, \overline{\boldsymbol{u}_{0}}\right\rangle\right) \\
& =1+\frac{4}{\sqrt{N}}+\frac{4}{N^{1 / 4}} \operatorname{Re}\left(\boldsymbol{u}_{0}, \boldsymbol{u}_{0}\right) \leq 1+\frac{4}{\sqrt{N}}+\frac{4}{N^{1 / 4}}\left|\left(\boldsymbol{u}_{0}, \boldsymbol{u}_{0}\right)\right| \\
& =1+\frac{4}{\sqrt{N}}+\frac{4 \delta_{0}^{2}}{N^{1 / 4}} \leq 1+\frac{4}{\sqrt{N}}+\frac{4}{\sqrt{N}} \leq 1+\frac{8}{\sqrt{N}} \leq \frac{7}{3} .
\end{aligned}
$$

Then (1) holds because of (2.8) and (2.9).

Finally, we prove (2). Let $p \in \bar{\varpi}_{j}$. Then we have

$$
\begin{aligned}
\left|\left(F_{j-1}(p), \boldsymbol{u}_{0}\right)\right| & =\left|\left(F_{j-1}(p)-F_{j-1}\left(\zeta_{j}\right), \boldsymbol{u}_{0}\right)+\left(F_{j-1}\left(\zeta_{j}\right), \boldsymbol{u}_{0}\right)\right| \\
& \leq\left|\left(F_{j-1}(p)-F_{j-1}\left(\zeta_{j}\right), \boldsymbol{u}_{0}\right)\right|+\left|\left(F_{j-1}\left(\zeta_{j}\right), \boldsymbol{u}_{0}\right)\right| \\
& \leq\left|F_{j-1}(p)-F_{j-1}\left(\zeta_{j}\right)\right|\left|\boldsymbol{u}_{0}\right|+\left|\left(\left|F_{j-1}\left(\zeta_{j}\right)\right| \boldsymbol{u}_{0}, \boldsymbol{u}_{0}\right)\right| \\
& \leq \frac{6 \mu}{N}+\left|F_{j-1}\left(\zeta_{j}\right)\right|\left|\left(\boldsymbol{u}_{0}, \boldsymbol{u}_{0}\right)\right| \\
& \leq \frac{6 \mu}{N}+\delta_{0}^{2}\left|F_{j-1}\left(\zeta_{j}\right)\right| \leq \frac{6 \mu}{N}+\frac{\left|F_{j-1}\left(\zeta_{j}\right)\right|}{N^{1 / 4}} \\
& =\left|F_{j-1}\left(\zeta_{j}\right)\right|\left(\frac{1}{N^{1 / 4}}+\frac{6 \mu}{N\left|F_{j-1}\left(\zeta_{j}\right)\right|}\right) \\
& \leq\left|F_{j-1}\left(\zeta_{j}\right)\right|\left(\frac{1}{N^{1 / 4}}+\frac{6 \mu}{\sqrt{N}}\right) \\
& =\left|F_{j-1}\left(\zeta_{j}\right)\right| \frac{1}{N^{1 / 4}}\left(1+\frac{6 \mu}{N^{1 / 4}}\right) \\
& \leq\left|F_{j-1}\left(\zeta_{j}\right)\right| \frac{1}{N^{1 / 4}}\left(1+\frac{6 \mu}{\sqrt{6}}\right)
\end{aligned}
$$


Thus we have

$$
\left|\left(F_{j-1}(p), \boldsymbol{u}_{0}\right)\right| \leq \frac{\left|F_{j-1}\left(\zeta_{j}\right)\right|}{N^{1 / 4}}(1+3 \mu) .
$$

On the other hand, since

$$
\frac{1}{\sqrt{1+x}} \geq 1-\frac{x}{2} \quad(0 \leq x \leq 2),
$$

we have

$$
\begin{aligned}
& \left|\left\langle\frac{F_{j-1}(p)}{\left|F_{j-1}(p)\right|}, \boldsymbol{u}\right\rangle\right| \\
& =\frac{1}{|\tilde{\boldsymbol{u}}|}\left|\left\langle\frac{F_{j-1}(p)}{\left|F_{j-1}(p)\right|}, \boldsymbol{u}_{0}\right\rangle+\frac{2}{N^{1 / 4}}\left\langle\frac{F_{j-1}(p)}{\left|F_{j-1}(p)\right|}, \overline{\boldsymbol{u}_{0}}\right\rangle\right| \\
& \geq \frac{1}{\sqrt{1+\frac{8}{\sqrt{N}}}}\left|\left\langle\frac{F_{j-1}(p)}{\left|F_{j-1}(p)\right|}, \boldsymbol{u}_{0}\right\rangle+\frac{2}{N^{1 / 4}}\left\langle\frac{F_{j-1}(p)}{\left|F_{j-1}(p)\right|}, \overline{\boldsymbol{u}_{0}}\right\rangle\right| \\
& \geq\left(1-\frac{4}{\sqrt{N}}\right)\left(\left|\left\langle\frac{F_{j-1}(p)}{\left|F_{j-1}(p)\right|}, \boldsymbol{u}_{0}\right\rangle\right|-\frac{2}{N^{1 / 4}}\left|\left\langle\frac{F_{j-1}(p)}{\left|F_{j-1}(p)\right|}, \overline{\boldsymbol{u}_{0}}\right\rangle\right|\right) \\
& \geq\left(1-\frac{4}{\sqrt{N}}\right)\left[\left(1-\frac{6 \mu^{2}}{\sqrt{N}}\right)-\frac{2}{N^{1 / 4}}\left|\left\langle\frac{F_{j-1}(p)}{\left|F_{j-1}(p)\right|}, \overline{\boldsymbol{u}_{0}}\right\rangle\right|\right] \\
& =\left(1-\frac{4}{\sqrt{N}}\right)\left[\left(1-\frac{6 \mu^{2}}{\sqrt{N}}\right)-\frac{2}{N^{1 / 4}}\left|\left(\frac{F_{j-1}(p)}{\left|F_{j-1}(p)\right|}, \boldsymbol{u}_{0}\right)\right|\right] \\
& \geq\left(1-\frac{4}{\sqrt{N}}\right)\left[\left(1-\frac{6 \mu^{2}}{\sqrt{N}}\right)-\frac{2\left|F_{j-1}\left(\zeta_{j}\right)\right|}{\sqrt{N}\left|F_{j-1}(p)\right|}(1+3 \mu)\right] \\
& \geq\left(1-\frac{4}{\sqrt{N}}\right)\left[\left(1-\frac{6 \mu^{2}}{\sqrt{N}}\right)-\frac{4}{\sqrt{N}}(1+3 \mu)\right] \\
& =\left(1-\frac{4}{\sqrt{N}}\right)\left(1-\frac{1}{\sqrt{N}}\left(6 \mu^{2}+12 \mu+4\right)\right) \\
& \geq 1-\frac{1}{\sqrt{N}}\left(6 \mu^{2}+12 \mu+8\right)+\frac{4}{N}\left(6 \mu^{2}+12 \mu+4\right) \\
& \geq 1-\frac{1}{\sqrt{N}}\left(6 \mu^{2}+12 \mu+8\right)=1-\frac{c_{1}}{\sqrt{N}} \text {. }
\end{aligned}
$$

Thus we have the conclusion. 


\section{The proof of the Key Lemma 1.4}

To continue the procedure of the iterational construction of $F_{j}$, we prepare the following lemma:

Lemma 3.1. For a unit vector $\boldsymbol{u} \in \mathbf{C}^{3}$, there exist $P \in \mathrm{SO}(3)$ and $\tau \in \mathbf{R}$ such that

$$
e^{-\mathrm{i} \tau} P \boldsymbol{u}=\left(\begin{array}{c}
0 \\
\mathrm{i} \sin \theta \\
\cos \theta
\end{array}\right) \quad(\mathrm{i}=\sqrt{-1})
$$

where we consider elements in $\mathbf{C}^{3}$ as column vectors (cf. Remark 1.1). Here, $\theta$ is a real number such that

$$
\cos 2 \theta=\cos ^{2} \theta-\sin ^{2} \theta=|(\boldsymbol{u}, \boldsymbol{u})| \quad\left(0 \leq \theta \leq \frac{\pi}{4}\right) .
$$

Proof. Write $\boldsymbol{u}=\boldsymbol{x}+\mathrm{i} \boldsymbol{y}\left(\boldsymbol{x}, \boldsymbol{y} \in \mathbf{R}^{3}\right)$, and let $\tau \in \mathbf{R}$ be

$$
\tau=\left\{\begin{array}{lc}
\frac{1}{2} \arctan \frac{2\langle\boldsymbol{x}, \boldsymbol{y}\rangle}{|\boldsymbol{x}|^{2}-|\boldsymbol{y}|^{2}} & (\text { when }|\boldsymbol{x}| \neq|\boldsymbol{y}|) \\
\frac{\pi}{4} & (\text { when }|\boldsymbol{x}|=|\boldsymbol{y}|)
\end{array}\right.
$$
Then $\hat{\boldsymbol{u}}:=e^{-\mathrm{i} \tau} \boldsymbol{u}$ satisfies $\langle\operatorname{Re} \hat{\boldsymbol{u}}, \operatorname{Im} \hat{\boldsymbol{u}}\rangle=0$. Moreover, replacing $\tau$ with $\tau+\frac{\pi}{2}$ if
necessary, we may assume

$$
|\operatorname{Re} \hat{\boldsymbol{u}}| \geq|\operatorname{Im} \hat{\boldsymbol{u}}|
$$

without loss of generality. In particular, since $|\hat{\boldsymbol{u}}|=1$, it holds that $|\operatorname{Re} \hat{\boldsymbol{u}}|>0$. Hence there exists a matrix $P_{1} \in \mathrm{SO}(3)$ such that

$$
P_{1}(\operatorname{Re} \hat{\boldsymbol{u}})=\left(\begin{array}{l}
0 \\
0 \\
t
\end{array}\right) \quad(t>0) .
$$

Since $P_{1}$ is a real matrix, $\operatorname{Im}\left(P_{1} \hat{\boldsymbol{u}}\right)$ is orthogonal to $\operatorname{Re}\left(P_{1} \hat{\boldsymbol{u}}\right)$. Hence, we have

$$
P_{1}(\hat{\boldsymbol{u}})=\left(\begin{array}{c}
\mathrm{i} u_{1} \\
\mathrm{i} u_{2} \\
t
\end{array}\right) \quad\left(u_{1}, u_{2}, t \in \mathbf{R}, t>0,\left(u_{1}\right)^{2}+\left(u_{2}\right)^{2}+t^{2}=1\right) .
$$

Moreover, $t \geq \sqrt{\left(u_{1}\right)^{2}+\left(u_{2}\right)^{2}}$ holds because of (3.2). Next, choose a real number $s$ such that

$$
\left(\begin{array}{cc}
\cos s & -\sin s \\
\sin s & \cos s
\end{array}\right)\left(\begin{array}{l}
u_{1} \\
u_{2}
\end{array}\right)=\left(\begin{array}{l}
0 \\
u
\end{array}\right), \quad \text { where } u:=\sqrt{\left(u_{1}\right)^{2}+\left(u_{2}\right)^{2}} \geq 0 .
$$


Set

$$
P:=\left(\begin{array}{ccc}
\cos s & -\sin s & 0 \\
\sin s & \cos s & 0 \\
0 & 0 & 1
\end{array}\right) \cdot P_{1} \in \mathrm{SO}(3)
$$

Then

$$
e^{-\mathrm{i} \tau} P \boldsymbol{u}=P \hat{\boldsymbol{u}}=\left(\begin{array}{c}
0 \\
\mathrm{i} u \\
t
\end{array}\right) \quad\left(u, t \in \mathbf{R}, t \geq u \geq 0, u^{2}+t^{2}=1\right)
$$

Hence there exists $\theta \in\left[0, \frac{\pi}{4}\right]$ such that $u=\sin \theta, t=\cos \theta . \quad$ In particular,

$$
|(\boldsymbol{u}, \boldsymbol{u})|=t^{2}-u^{2}=\cos ^{2} \theta-\sin ^{2} \theta=\cos 2 \theta
$$

holds and thus we have the conclusion.

We set

$$
A:=\left(\begin{array}{ccc}
\sqrt{\cos 2 x} & 0 & 0 \\
0 & \cos x & -\mathrm{i} \sin x \\
0 & \mathrm{i} \sin x & \cos x
\end{array}\right)
$$

where $x \in\left[0, \frac{\pi}{4}\right]$. Then $A$ is non-singular if and only if $x \neq \frac{\pi}{4}$. In this case,

$$
A \in \sqrt{\cos 2 x} \cdot \mathrm{O}(3, \mathbf{C}),
$$

and

$$
A^{-1}=\frac{1}{\cos 2 x}\left(\begin{array}{ccc}
\sqrt{\cos 2 x} & 0 & 0 \\
0 & \cos x & \mathrm{i} \sin x \\
0 & -\mathrm{i} \sin x & \cos x
\end{array}\right)
$$
satisfies

Lemma 3.2. Let $x \in\left[0, \frac{\pi}{4}\right)$ be a real number. Then the matrix $A$ in (3.3)
fies

$$
\|A\|=\cos x+\sin x \leq \sqrt{2}, \quad\left\|A^{-1}\right\|=\frac{\cos x+\sin x}{\cos 2 x} \leq \frac{\sqrt{2}}{\cos 2 x} .
$$

Proof. Since the eigenvalues of the matrix

$$
A^{*} A=\left(\begin{array}{ccc}
\cos 2 x & 0 & 0 \\
0 & 1 & -\mathrm{i} \sin 2 x \\
0 & \mathrm{i} \sin 2 x & 1
\end{array}\right)
$$


are $(\cos x-\sin x)^{2}, \cos 2 x$, and $(\cos x+\sin x)^{2},(1.5)$ implies that $\|A\|=\cos x+$ $\sin x=\sqrt{2} \sin \left(x+\frac{\pi}{4}\right) \leq \sqrt{2}$. On the other hand, the eigenvalues of $\left(A^{-1}\right)^{*} A^{-1}$
are $\frac{(\cos x-\sin x)^{2}}{\cos ^{2} 2 x}, \frac{1}{\cos 2 x}$, and $\quad \frac{(\cos x+\sin x)^{2}}{\cos ^{2} 2 x}$.

Hence

$$
\left\|A^{-1}\right\|=\frac{\cos x+\sin x}{\cos 2 x} \leq \frac{\sqrt{2}}{\cos 2 x}
$$

which is the conclusion.

We return to the construction of $F_{j}$ : Take $\boldsymbol{u}$ as in Lemma 2.4, and take $P \in \mathrm{SO}(3)$ and $\tau \in \mathbf{R}$ as in Lemma 3.1, where $\theta \in\left[0, \frac{\pi}{4}\right]$ is given by $\cos 2 \theta=$ $|(\boldsymbol{u}, \boldsymbol{u})|$. Observe that by (1) of Lemma 2.4 we have

$$
\delta:=\sqrt{\cos 2 \theta} \geq \frac{1}{N^{1 / 8}}
$$

and therefore $\theta \in\left[0, \frac{\pi}{4}\right)$. We set

$$
F:=e^{\mathrm{i} \tau} P F_{j-1}, \quad \varphi:=\varphi_{F}=\frac{d F}{d z}=e^{\mathrm{i} \tau} P \varphi_{j-1} .
$$

Since $P \in \mathrm{SO}(3) \subset \mathrm{O}(3, \mathrm{C}), F$ is a holomorphic null immersion. On the other hand, since $P \in \mathrm{SO}(3) \subset \mathrm{U}(3), F$ is congruent to $F_{j-1}$ in $\mathbf{C}^{3}$. In particular,

$$
|\varphi|=\left|\varphi_{j-1}\right|, \quad|\varphi(q)-\varphi(p)|=\left|\varphi_{j-1}(q)-\varphi_{j-1}(p)\right|
$$

hold for $p, q \in \overline{\mathbf{D}}_{1}$.

Taking into account (3.6), we consider the matrix

$$
A=\left(\boldsymbol{a}^{(1)}, \boldsymbol{a}^{(2)}, \boldsymbol{a}^{(3)}\right):=\left(\begin{array}{ccc}
\delta & 0 & 0 \\
0 & \cos \theta & -\mathrm{i} \sin \theta \\
0 & \mathrm{i} \sin \theta & \cos \theta
\end{array}\right) \in \delta \cdot \mathrm{O}(3, \mathbf{C}) .
$$

In particular, by (3.1), it holds that

$$
\boldsymbol{a}^{(3)}=\overline{e^{-\mathrm{i} \tau} P \boldsymbol{u}}=e^{\mathrm{i} \tau} P \overline{\boldsymbol{u}} .
$$

By Lemma 3.2 and (3.6), it holds that

$$
\|A\| \leq \sqrt{2}, \quad\left\|A^{-1}\right\| \leq \frac{\sqrt{2}}{\delta^{2}} \leq \sqrt{2} N^{1 / 4} .
$$


Using the matrix $A$ in (3.9), we set

$$
\begin{aligned}
& E=\left(E^{(1)}, E^{(2)}, E^{(3)}\right)^{t}:=A^{-1} F=e^{\mathrm{i} \tau} A^{-1} P F_{j-1}, \\
& \psi:=\frac{d E}{d z}=A^{-1} \varphi=e^{\mathrm{i} \tau} A^{-1} P \varphi_{j-1} .
\end{aligned}
$$

Since $A \in \delta \cdot \mathrm{O}(3, \mathbf{C}), E$ is a holomorphic null immersion although it is not necessarily congruent to $F_{j-1}$. Moreover, by (3.12), (1.4), (3.11) and (3.8), we have

$$
\begin{aligned}
|\psi| & =\left|A^{-1} \varphi\right| \geq \frac{1}{\|A\|}|\varphi| \geq \frac{|\varphi|}{\sqrt{2}}=\frac{\left|\varphi_{j-1}\right|}{\sqrt{2}} \\
|\psi(q)-\psi(p)| & =\left|A^{-1}(\varphi(q)-\varphi(p))\right| \leq\left\|A^{-1}\right\||\varphi(q)-\varphi(p)| \\
& \leq \sqrt{2} N^{1 / 4}\left|\varphi_{j-1}(q)-\varphi_{j-1}(p)\right| .
\end{aligned}
$$

Lemma 3.3. Let $G=G_{E}: \overline{\mathbf{D}}_{1} \rightarrow S^{2}$ be the Gauss map of $E$ as in (3.12) (cf. (1.18)). Then there exists a real matrix $Q$

$$
Q=\left(\begin{array}{ccc}
1 & 0 & 0 \\
0 & \cos \Theta & -\sin \Theta \\
0 & \sin \Theta & \cos \Theta
\end{array}\right) \in \operatorname{SO}(3), \quad|\Theta| \leq \frac{4}{\sqrt{N}}
$$

such that

$$
\operatorname{dist}_{S^{2}}\left(Q G(p), \pm \boldsymbol{e}_{3}\right) \geq \frac{1}{\sqrt{N}} \quad\left(\boldsymbol{e}_{3}:=\left(\begin{array}{l}
0 \\
0 \\
1
\end{array}\right)\right)
$$

holds for each point $p \in \bar{\varpi}_{j}$, where dist $_{S^{2}}$ is the canonical distance function of the unit sphere $S^{2}$ and $G(p) \in \mathbf{R}^{3}$ is considered as a column vector (cf. Remark 1.1). In particular, as in (3.9), one has:

$$
\text { the matrix } A \text { commutes with } Q^{-1} \text {, }
$$

and

$$
\left\|Q^{-1}-\mathrm{id}\right\| \leq|\Theta| \leq \frac{4}{\sqrt{N}}
$$

holds.

Proof. By (3.9) and (3.15), the equality (3.17) is trivial. Moreover, since

$$
Q^{-1}-\mathrm{id}=\left(\begin{array}{ccc}
0 & 0 & 0 \\
0 & \cos \Theta-1 & \sin \Theta \\
0 & -\sin \Theta & \cos \Theta-1
\end{array}\right)=-2 \sin \frac{\Theta}{2}\left(\begin{array}{ccc}
0 & 0 & 0 \\
0 & \sin \frac{\Theta}{2} & -\cos \frac{\Theta}{2} \\
0 & \cos \frac{\Theta}{2} & \sin \frac{\Theta}{2}
\end{array}\right),
$$


the maximum eigenvalue of $\left(Q^{-1}-\mathrm{id}\right)^{*}\left(Q^{-1}-\mathrm{id}\right)$ is $\left(2 \sin \frac{\Theta}{2}\right)^{2}$. Hence by $(1.5)$,

$$
\left\|Q^{-1}-\mathrm{id}\right\|=2\left|\sin \frac{\Theta}{2}\right| \leq|\Theta| .
$$

holds, and thus we have (3.18).

So, to prove Lemma 3.3 , it is sufficient to show (3.16) for suitable $Q$. The Euclidean distance between $G(p)$ and $G\left(\zeta_{j}\right)$ in $\mathbf{R}^{3}$ can be estimated as

$$
\begin{aligned}
& \left|G(p)-G\left(\zeta_{j}\right)\right|=\left|\frac{\psi(p) \times \overline{\psi(p)}}{|\psi(p)|^{2}}-\frac{\psi\left(\zeta_{j}\right) \times \overline{\psi\left(\zeta_{j}\right)}}{\left|\psi\left(\zeta_{j}\right)\right|^{2}}\right| \quad(\text { by (1.18)) } \\
& =\left.\frac{1}{|\psi(p)|^{2}\left|\psi\left(\zeta_{j}\right)\right|^{2}}|(\psi(p) \times \overline{\psi(p)})| \psi\left(\zeta_{j}\right)\right|^{2}-\left(\psi\left(\zeta_{j}\right) \times \overline{\psi\left(\zeta_{j}\right)}\right)|\psi(p)|^{2} \mid \\
& =\left.\frac{1}{|\psi(p)|^{2}\left|\psi\left(\zeta_{j}\right)\right|^{2}}|(\psi(p) \times \overline{\psi(p)})| \psi\left(\zeta_{j}\right)\right|^{2}-(\psi(p) \times \overline{\psi(p)})|\psi(p)|^{2} \\
& +(\psi(p) \times \overline{\psi(p)})|\psi(p)|^{2}-\left(\psi\left(\zeta_{j}\right) \times \overline{\psi\left(\zeta_{j}\right)}\right)|\psi(p)|^{2} \mid \\
& =\frac{1}{|\psi(p)|^{2}\left|\psi\left(\zeta_{j}\right)\right|^{2}} \mid(\psi(p) \times \overline{\psi(p)})\left(\left|\psi\left(\zeta_{j}\right)\right|^{2}-|\psi(p)|^{2}\right) \\
& +|\psi(p)|^{2}\left(\psi(p) \times \overline{\psi(p)}-\psi\left(\zeta_{j}\right) \times \overline{\psi\left(\zeta_{j}\right)}\right) \mid \\
& \leq \frac{|\psi(p)|^{2}\left(\left.|| \psi\left(\zeta_{j}\right)\right|^{2}-|\psi(p)|^{2}|+| \psi(p) \times \overline{\psi(p)}-\psi\left(\zeta_{j}\right) \times \overline{\psi\left(\zeta_{j}\right)} \mid\right)}{|\psi(p)|^{2}\left|\psi\left(\zeta_{j}\right)\right|^{2}} \\
& =\frac{1}{\left|\psi\left(\zeta_{j}\right)\right|^{2}}\left(|| \psi\left(\zeta_{j}\right)|-| \psi(p)||\left(\left|\psi\left(\zeta_{j}\right)\right|+|\psi(p)|\right)\right. \\
& \left.+\left|\psi(p) \times \overline{\psi(p)}-\psi(p) \times \overline{\psi\left(\zeta_{j}\right)}+\psi(p) \times \overline{\psi\left(\zeta_{j}\right)}-\psi\left(\zeta_{j}\right) \times \overline{\psi\left(\zeta_{j}\right)}\right|\right) \\
& \leq \frac{1}{\left|\psi\left(\zeta_{j}\right)\right|^{2}}\left(\left|\psi\left(\zeta_{j}\right)-\psi(p)\right|\left(\left|\psi\left(\zeta_{j}\right)\right|+|\psi(p)|\right)\right. \\
& \left.+\left|\psi(p) \times\left(\overline{\psi(p)-\psi\left(\zeta_{j}\right)}\right)+\left(\psi(p)-\psi\left(\zeta_{j}\right)\right) \times \overline{\psi\left(\zeta_{j}\right)}\right|\right) \\
& \leq \frac{2}{\left|\psi\left(\zeta_{j}\right)\right|^{2}}\left|\psi(p)-\psi\left(\zeta_{j}\right)\right|\left(\left|\psi(p)-\psi\left(\zeta_{j}\right)\right|+2\left|\psi\left(\zeta_{j}\right)\right|\right) \\
& \leq \frac{2}{\left|\psi\left(\zeta_{j}\right)\right|}\left|\psi(p)-\psi\left(\zeta_{j}\right)\right|\left(2+\frac{\left|\psi(p)-\psi\left(\zeta_{j}\right)\right|}{\left|\psi\left(\zeta_{j}\right)\right|}\right) \\
& \leq \frac{2 \sqrt{2}}{\left|\varphi_{j-1}\left(\zeta_{j}\right)\right|}\left|\psi(p)-\psi\left(\zeta_{j}\right)\right|\left(2+\frac{\sqrt{2}\left|\psi(p)-\psi\left(\zeta_{j}\right)\right|}{\left|\varphi_{j-1}\left(\zeta_{j}\right)\right|}\right)
\end{aligned}
$$




$$
\begin{aligned}
& \leq \frac{4 N^{1 / 4}\left|\varphi_{j-1}(p)-\varphi_{j-1}\left(\zeta_{j}\right)\right|}{\left|\varphi_{j-1}\left(\zeta_{j}\right)\right|}\left(2+\frac{2 N^{1 / 4}\left|\varphi_{j-1}(p)-\varphi_{j-1}\left(\zeta_{j}\right)\right|}{\left|\varphi_{j-1}\left(\zeta_{j}\right)\right|}\right) \\
& \leq \frac{8 N^{1 / 4}\left|\varphi_{j-1}(p)-\varphi_{j-1}\left(\zeta_{j}\right)\right|}{v}\left(2+\frac{4 N^{1 / 4}}{v}\left|\varphi_{j-1}(p)-\varphi_{j-1}\left(\zeta_{j}\right)\right|\right) \\
& \leq \frac{8 N^{1 / 4}}{v} \frac{6 \mu+2 \varepsilon}{N}\left(2+\frac{4 N^{1 / 4}}{v} \frac{6 \mu+2 \varepsilon}{N}\right) \\
& =\frac{1}{\sqrt{N}} \frac{1}{N^{1 / 4}}\left(\frac{16(3 \mu+\varepsilon)}{v}\left(2+\frac{4(6 \mu+2 \varepsilon)}{N^{3 / 4} v}\right)\right) \\
& \leq \frac{1}{\sqrt{N}} \frac{1}{N^{1 / 4}}\left(\frac{16(3 \mu+\varepsilon)}{v}\left(2+\frac{4(6 \mu+2 \varepsilon)}{\sqrt{6}^{3} v}\right)\right) \\
& \leq \frac{1}{\sqrt{N}} \frac{1}{N^{1 / 4}}\left(\frac{16(3 \mu+\varepsilon)}{v}\left(2+\frac{6 \mu+2 \varepsilon}{3 v}\right)\right) \leq \frac{1}{2 \sqrt{N}}
\end{aligned}
$$

Then we have

$$
\begin{aligned}
\operatorname{dist}_{S^{2}}\left(G(p), G\left(\zeta_{j}\right)\right) & =2 \arcsin \left(\frac{1}{2}\left|G(p)-G\left(\zeta_{j}\right)\right|\right) \\
& \leq \frac{\pi}{2}\left|G(p)-G\left(\zeta_{j}\right)\right| \leq 2\left|G(p)-G\left(\zeta_{j}\right)\right| \leq \frac{1}{\sqrt{N}}
\end{aligned}
$$

Here we used the inequality $\arcsin x \leq \pi x / 2(0 \leq x \leq 1)$. In particular, $G\left(\varpi_{j}\right)$ is contained in the geodesic disc in the unit sphere $S^{2}$ centered at $G\left(\zeta_{j}\right)$ with radius $1 / \sqrt{N}$.

CASE 1. Assume both $\operatorname{dist}_{S^{2}}\left(G\left(\zeta_{j}\right), \boldsymbol{e}_{3}\right) \geq 2 / \sqrt{N}$ and $\operatorname{dist}_{S^{2}}\left(G\left(\zeta_{j}\right),-\boldsymbol{e}_{3}\right) \geq$ $2 / \sqrt{N}$ hold, where $\boldsymbol{e}_{3}=(0,0,1)$. Then for each $p \in \bar{\varpi}_{j}$, (3.19) implies that

$$
\begin{aligned}
\operatorname{dist}_{S^{2}}\left(G(p), \boldsymbol{e}_{3}\right) & \geq \operatorname{dist}_{S^{2}}\left(G\left(\zeta_{j}\right), \boldsymbol{e}_{3}\right)-\operatorname{dist}_{S^{2}}\left(G(p), G\left(\zeta_{j}\right)\right) \\
& \geq \frac{2}{\sqrt{N}}-\operatorname{dist}_{S^{2}}\left(G(p), G\left(\zeta_{j}\right)\right) \geq \frac{2}{\sqrt{N}}-\frac{1}{\sqrt{N}}=\frac{1}{\sqrt{N}} .
\end{aligned}
$$

Similarly $\operatorname{dist}_{S^{2}}\left(G(p),-\boldsymbol{e}_{3}\right) \geq 1 / \sqrt{N}$ holds. Then we have the conclusion (3.16) for $Q=\mathrm{id}$ and $\Theta=0$.

\section{CASE 2. Assume}

$$
\operatorname{dist}_{S^{2}}\left(G\left(\zeta_{j}\right), \boldsymbol{e}_{3}\right)<\frac{2}{\sqrt{N}} .
$$


In this case, take the matrix $Q$ as in (3.15) with

$$
\Theta:=\frac{4}{\sqrt{N}} \text {. }
$$

Then

$$
\begin{aligned}
\operatorname{dist}_{S^{2}} & \left(Q G(p), \boldsymbol{e}_{3}\right) \\
& \geq \operatorname{dist}_{S^{2}}\left(Q \boldsymbol{e}_{3}, \boldsymbol{e}_{3}\right)-\operatorname{dist}_{S^{2}}\left(Q G(p), Q G\left(\zeta_{j}\right)\right)-\operatorname{dist}_{S^{2}}\left(Q G\left(\zeta_{j}\right), Q \boldsymbol{e}_{3}\right) \\
& =\frac{4}{\sqrt{N}}-\operatorname{dist}_{S^{2}}\left(Q G(p), Q G\left(\zeta_{j}\right)\right)-\operatorname{dist}_{S^{2}}\left(Q G\left(\zeta_{j}\right), Q \boldsymbol{e}_{3}\right) \quad(\text { by }(3.21)) \\
& =\frac{4}{\sqrt{N}}-\operatorname{dist}_{S^{2}}\left(G(p), G\left(\zeta_{j}\right)\right)-\operatorname{dist}_{S^{2}}\left(G\left(\zeta_{j}\right), \boldsymbol{e}_{3}\right) \quad(Q \in \mathrm{SO}(3)) \\
& \geq \frac{4}{\sqrt{N}}-\frac{1}{\sqrt{N}}-\operatorname{dist}_{S^{2}}\left(G\left(\zeta_{j}\right), \boldsymbol{e}_{3}\right)>\frac{1}{\sqrt{N}} \quad \text { (by (3.19), (3.20)). }
\end{aligned}
$$

On the other hand,

$$
\begin{array}{rlr}
\operatorname{dist}_{S^{2}} & \left(Q G(p), \boldsymbol{e}_{3}\right) \\
& \leq \operatorname{dist}_{S^{2}}\left(Q G(p), Q G\left(\zeta_{j}\right)\right)+\operatorname{dist}_{S^{2}}\left(Q G\left(\zeta_{j}\right), Q \boldsymbol{e}_{3}\right)+\operatorname{dist}_{S^{2}}\left(Q \boldsymbol{e}_{3}, \boldsymbol{e}_{3}\right) \\
& =\operatorname{dist}_{S^{2}}\left(Q G(p), Q G\left(\zeta_{j}\right)\right)+\operatorname{dist}_{S^{2}}\left(Q G\left(\zeta_{j}\right), Q \boldsymbol{e}_{3}\right)+\frac{4}{\sqrt{N}} & (\text { by }(3.21)) \\
& =\operatorname{dist}_{S^{2}}\left(G(p), G\left(\zeta_{j}\right)\right)+\operatorname{dist}_{S^{2}}\left(G\left(\zeta_{j}\right), \boldsymbol{e}_{3}\right)+\frac{4}{\sqrt{N}} & (Q \in \mathrm{SO}(3)) \\
& \leq \frac{1}{\sqrt{N}}+\operatorname{dist}_{S^{2}}\left(G\left(\zeta_{j}\right), \boldsymbol{e}_{3}\right)+\frac{4}{\sqrt{N}} & (\text { by }(3.19)) \\
& <\frac{1}{\sqrt{N}}+\frac{2}{\sqrt{N}}+\frac{4}{\sqrt{N}}=\frac{7}{\sqrt{N}} & \text { (by }(3.20))
\end{array}
$$

and then,

$$
\operatorname{dist}_{S^{2}}\left(Q G(p),-\boldsymbol{e}_{3}\right)=\pi-\operatorname{dist}_{S^{2}}\left(Q G(p), \boldsymbol{e}_{3}\right) \geq 3-\frac{7}{6} \geq \frac{1}{\sqrt{N}}
$$

because of (1.13). Thus, we have the conclusion (3.16).

CASE 3. If $\operatorname{dist}_{S^{2}}\left(G\left(\zeta_{j}\right),-\boldsymbol{e}_{3}\right)<2 / \sqrt{N}$ holds, then we have the conclusion by the same way as in the previous case.

Using $P \in \mathrm{SO}(3), \tau \in \mathbf{R}$ in (3.1), $A \in \delta \cdot \mathrm{O}(3, \mathbf{C})$ in (3.9) and $Q \in \mathrm{SO}(3)$ in (3.15), we define

$$
\tilde{E}:=Q E=B^{-1} F_{j-1}, \quad \tilde{\psi}:=\frac{d \tilde{E}}{d z}=Q \psi
$$


where

$$
B=\left(\boldsymbol{b}^{(1)}, \boldsymbol{b}^{(2)}, \boldsymbol{b}^{(3)}\right):=\left(e^{\mathrm{i} \tau} Q A^{-1} P\right)^{-1} \in\left(e^{-\mathrm{i} \tau} \delta\right) \cdot \mathrm{O}(3, \mathbf{C}) .
$$

Here, the elements of $\mathbf{C}^{3}$ are considered as column vectors (cf. Remark 1.1). Then $\tilde{E}$ is a holomorphic null immersion which is congruent to $E$ in (3.12). Denote by $(g, \eta)$ the Weierstrass data $($ cf. $(1.8))$ of $\tilde{E}$ :

$$
\tilde{\psi}=\frac{1}{2}\left(1-g^{2}, \mathrm{i}\left(1+g^{2}\right), 2 g\right) \eta, \quad|\tilde{\psi}|^{2}=\frac{1}{2}\left(1+|g|^{2}\right)^{2}|\eta|^{2} .
$$

Then we have

LEMma 3.4. The meromorphic function $g$ as in (3.24) satisfies

$$
\frac{1}{2 \sqrt{N}} \leq|g| \leq 2 \sqrt{N} \quad \text { and } \quad \frac{|g|}{1+|g|^{2}} \geq \frac{2 \sqrt{N}}{1+4 N} \quad\left(\text { on } \bar{\varpi}_{j}\right) \text {. }
$$

Proof. The Gauss map $\tilde{G}$ of $\tilde{E}$ is obtained by

$$
\tilde{G}=Q G=\frac{1}{1+|g|^{2}}\left(\begin{array}{c}
2 \operatorname{Re} g \\
2 \operatorname{Im} g \\
|g|^{2}-1
\end{array}\right) \text {. }
$$

Here, since $\tilde{G}=Q G$ satisfies $(3.16)$ on $\bar{\varpi}_{j}$, it holds that

$$
\begin{gathered}
\operatorname{dist}_{S^{2}}\left(\tilde{\boldsymbol{G}}, \boldsymbol{e}_{3}\right)=\arccos \left(\tilde{\boldsymbol{G}} \cdot \boldsymbol{e}_{3}\right)=\arccos \left(\frac{|g|^{2}-1}{|g|^{2}+1}\right) \geq \frac{1}{\sqrt{N}}, \\
\operatorname{dist}_{S^{2}}\left(\tilde{\boldsymbol{G}},-\boldsymbol{e}_{3}\right)=\arccos \left(\tilde{\boldsymbol{G}} \cdot\left(-\boldsymbol{e}_{3}\right)\right)=\arccos \left(\frac{1-|g|^{2}}{|g|^{2}+1}\right) \geq \frac{1}{\sqrt{N}}
\end{gathered}
$$

on $\bar{\varpi}_{j}$, where "." denotes the canonical inner product of $\mathbf{R}^{3}$. Since (3.25) implies

we have

$$
\frac{|g|^{2}-1}{|g|^{2}+1} \leq \cos \frac{1}{\sqrt{N}}
$$

$$
|g|^{2} \leq \frac{1+\cos \frac{1}{\sqrt{N}}}{1-\cos \frac{1}{\sqrt{N}}}=\cot ^{2} \frac{1}{2 \sqrt{N}} \leq(2 \sqrt{N})^{2}
$$

Similarly, by (3.26), we have

$$
|g|^{2} \geq \tan ^{2} \frac{1}{2 \sqrt{N}} \geq\left(\frac{1}{2 \sqrt{N}}\right)^{2}
$$

Thus, we have the first inequality of the conclusion. The second inequality is obtained immediately by the first inequality. 
We set

$$
\boldsymbol{v}_{j}:=\overline{\boldsymbol{b}^{(3)}}
$$

where $\boldsymbol{b}^{(3)}$ is the third column of the matrix $B$ as in (3.23).

LEMMA 3.5. The vector $\boldsymbol{v}_{j}$ in (3.27) is a unit vector satisfying $\left|\left(\boldsymbol{v}_{j}, \boldsymbol{v}_{j}\right)\right| \geq$ $1 / N^{1 / 4}$. Moreover, when (2.1) holds, that is, $\left|F_{j-1}\left(\zeta_{j}\right)\right| \geq 1 / \sqrt{N}$, it holds that

$$
\left|\left\langle\frac{F_{j-1}(p)}{\left|F_{j-1}(p)\right|}, \boldsymbol{v}_{j}\right\rangle\right| \geq 1-\frac{C_{2}}{\sqrt{N}} \text { for } p \in \bar{\varpi}_{j}
$$

where $C_{2}$ is the constant in (1.11).

Proof. Let $\boldsymbol{e}_{3}=(0,0,1)$. Since the matrix $A$ and $Q^{-1}$ commute (cf. (3.17)), the third column of the matrix $B$ is obtained as

$$
\begin{array}{rlrl}
\boldsymbol{b}^{(3)} & =B \boldsymbol{e}_{3}=e^{-\mathrm{i} \tau} P^{-1} A Q^{-1} \boldsymbol{e}_{3}=e^{-\mathrm{i} \tau} P^{-1} Q^{-1} A \boldsymbol{e}_{3} & & \text { (by }(3.23),(3.17)) \\
& =e^{-\mathrm{i} \tau} P^{-1} Q^{-1} \boldsymbol{a}^{(3)}=e^{-\mathrm{i} \tau} P^{-1} Q^{-1}\left(e^{\mathrm{i} \tau} P \overline{\boldsymbol{u}}\right) & & \text { (by }(3.9),(3.10)) \\
& =P^{-1} Q^{-1} P \overline{\boldsymbol{u}} .
\end{array}
$$

Taking into account that $P$ and $Q$ are real matrices, (3.27) implies that $\boldsymbol{v}_{j}=$ $P^{-1} Q^{-1} P \boldsymbol{u}$. Then by Lemma 2.4 , we have $\left|\boldsymbol{v}_{j}\right|=1,\left|\left(\boldsymbol{v}_{j}, \boldsymbol{v}_{j}\right)\right| \geq 1 / N^{1 / 4}$, because $P$, $Q \in \mathrm{SO}(3)$. Moreover, when $\left|F_{j-1}\left(\zeta_{j}\right)\right| \geq 1 / \sqrt{N}$ (i.e. (2.1) holds),

$$
\begin{aligned}
& \left|\left\langle\frac{F_{j-1}(p)}{\left|F_{j-1}(p)\right|}, \boldsymbol{v}_{j}\right\rangle\right| \\
& \quad=\left|\left\langle\frac{F_{j-1}(p)}{\left|F_{j-1}(p)\right|}, P^{-1} Q^{-1} P \boldsymbol{u}\right\rangle\right| \\
& =\left|\left\langle\frac{F_{j-1}(p)}{\left|F_{j-1}(p)\right|}, \boldsymbol{u}+P^{-1}\left(Q^{-1}-\mathrm{id}\right) P \boldsymbol{u}\right\rangle\right| \\
& \geq\left|\left\langle\frac{F_{j-1}(p)}{\left|F_{j-1}(p)\right|}, \boldsymbol{u}\right\rangle\right|-\left|\left\langle\frac{F_{j-1}(p)}{\left|F_{j-1}(p)\right|}, P^{-1}\left(Q^{-1}-\mathrm{id}\right) P \boldsymbol{u}\right\rangle\right| \\
& \geq\left|\left\langle\frac{F_{j-1}(p)}{\left|F_{j-1}(p)\right|}, \boldsymbol{u}\right\rangle\right|-\left|\frac{F_{j-1}(p)}{\left|F_{j-1}(p)\right|}\right| \cdot\left\|P^{-1}\left(Q^{-1}-\mathrm{id}\right) P\right\||\boldsymbol{u}| \quad(\text { by } \quad(1.4)) \\
& =\left|\left\langle\frac{F_{j-1}(p)}{\left|F_{j-1}(p)\right|}, \boldsymbol{u}\right\rangle\right|-\left\|P^{-1}\left(Q^{-1}-\mathrm{id}\right) P\right\| \\
& =\left|\left\langle\frac{F_{j-1}(p)}{\left|F_{j-1}(p)\right|}, \boldsymbol{u}\right\rangle\right|-\left\|Q^{-1}-\mathrm{id}\right\| \quad \quad(P \in \operatorname{SO}(3))
\end{aligned}
$$




$$
\begin{array}{ll}
\geq\left|\left\langle\frac{F_{j-1}(p)}{\left|F_{j-1}(p)\right|}, \boldsymbol{u}\right\rangle\right|-|\Theta| \geq\left|\left\langle\frac{F_{j-1}(p)}{\left|F_{j-1}(p)\right|}, \boldsymbol{u}\right\rangle\right|-\frac{4}{\sqrt{N}} & \text { (Lemma 3.3) } \\
\geq 1-\frac{c_{1}}{\sqrt{N}}-\frac{4}{\sqrt{N}}=1-\frac{C_{2}}{\sqrt{N}} . & \text { (Lemma 2.4, (1.11)). }
\end{array}
$$

Thus we have the conclusion.

Now, we apply the "López-Ros deformation" to the holomorphic null immersion $\tilde{E}$. The following lemma is the straightforward conclusion of the classical Runge's theorem:

Lemma 3.6. There exists a holomorphic function $h$ on $\mathbf{C}$ which does not vanish on $\mathbf{C}$ and satisfies

$$
\begin{cases}|h-1| \leq \varepsilon_{1} & \left(\text { on } \overline{\mathbf{D}}_{1} \backslash \varpi_{j}\right) \\ |h-T| \leq 1 & \left(\text { on } \omega_{j}\right)\end{cases}
$$

where

$$
\varepsilon_{1}=\frac{\varepsilon}{\varepsilon+4 \sqrt{2} \mu_{j-1} N^{9 / 4}}, \quad \mu_{j-1}=\max _{\overline{\mathbf{D}}_{1}}\left|\varphi_{j-1}\right|, \quad T=4 N^{7 / 2}+1 .
$$

Using the function $h$ in Lemma 3.6 as a López-Ros parameter, we produce new Weierstrass data as follows:

$$
\hat{g}:=\frac{g}{h}, \quad \hat{\eta}:=h \eta, \quad \hat{\psi}:=\frac{1}{2}\left(1-\hat{g}^{2}, \mathrm{i}\left(1+\hat{g}^{2}\right), 2 \hat{g}\right) \hat{\eta} .
$$

We denote

$$
\hat{E}(z):=\int_{0}^{z} \hat{\psi}(z) d z, \quad F_{j}:=B \hat{E}
$$

where $B$ is the matrix as in (3.23). By definition (3.29), $g \eta=\hat{g} \hat{\eta}$ holds. Thus, if we write

$$
\tilde{\psi}=\left(\tilde{\psi}^{(1)}, \tilde{\psi}^{(2)}, \tilde{\psi}^{(3)}\right) \quad \text { and } \quad \hat{\psi}=\left(\hat{\psi}^{(1)}, \hat{\psi}^{(2)}, \hat{\psi}^{(3)}\right)
$$

then

$$
\tilde{\psi}^{(3)}=\hat{\psi}^{(3)}
$$

holds.

By (3.30), the construction procedure of $F_{j}$ is accomplished. Thus, we obtain a sequence $\left\{F_{j}\right\}_{j=0,1, \ldots, 2 N}$ of holomorphic null immersions and a sequence $\left\{\boldsymbol{v}_{j}\right\}_{j=1, \ldots, 2 N}$ of unit vectors.

Now, we shall prove that $\left\{F_{j}\right\}$ and $\left\{\boldsymbol{v}_{j}\right\}$ satisfy the conclusions $(\mathrm{K}-0)-(\mathrm{K}-6)$ of the Key Lemma 1.4. 
Lemma $3.7((\mathrm{~K}-6))$. For each $j=1, \ldots, 2 N,\left\langle F_{j}, \boldsymbol{v}_{j}\right\rangle=\left\langle F_{j-1}, \boldsymbol{v}_{j}\right\rangle$ holds.

Proof. By (3.31), we have

$$
\left(\hat{E}, \boldsymbol{e}_{3}\right)=\int_{0}^{z} \hat{\psi}^{(3)}(w) d w=\int_{0}^{z} \tilde{\psi}^{(3)}(w) d w=\left(\tilde{E}, \boldsymbol{e}_{3}\right) \quad\left(\boldsymbol{e}_{3}=(0,0,1)\right) .
$$

Let $B$ be as in (3.23). Since $B \in\left(e^{-\mathrm{i} \tau} \delta\right) \cdot \mathrm{O}(3, \mathbf{C})$,

$$
(B \boldsymbol{x}, B \boldsymbol{y})=e^{-2 \mathrm{i} \tau} \delta^{2}(\boldsymbol{x}, \boldsymbol{y})
$$

holds. Then

$$
\begin{aligned}
\left\langle F_{j}, \boldsymbol{v}_{j}\right\rangle & =\left(F_{j}, \overline{\boldsymbol{v}}_{j}\right) \\
& =\left(B \hat{E}, \overline{\boldsymbol{v}}_{j}\right)=\left(B \hat{E}, \boldsymbol{b}^{(3)}\right)=\left(B \hat{E}, B \boldsymbol{e}_{3}\right) \quad(\text { by }(3.30),(3.27),(3.23)) \\
& =e^{-2 \mathrm{i} \tau} \delta^{2}\left(\hat{E}, \boldsymbol{e}_{3}\right)=e^{-2 \mathrm{i} \tau} \delta^{2}\left(\tilde{E}, \boldsymbol{e}_{3}\right)=\left\langle F_{j-1}, \boldsymbol{v}_{j}\right\rangle \quad(\text { by }(3.33),(3.32)) .
\end{aligned}
$$

The properties (K-4), (K-5) and (K-6) in the Key Lemma 1.4 for $l=j$ hold by Lemmas 3.5 and 3.7. The property (K-1) holds trivially because of (3.30). So we shall prove that $F_{j}(j=1, \ldots, 2 N)$ satisfies $(\mathrm{K}-2),(\mathrm{K}-3)$ of the Key Lemma 1.4 .

LEMMA 3.8. The holomorphic null immersion $F_{j}$ as in (3.30) satisfies

$$
\begin{array}{rlrl}
\left|\varphi_{j}-\varphi_{j-1}\right| & \leq \frac{\varepsilon}{2 N^{2}} \quad \text { on } \overline{\mathbf{D}}_{1} \backslash \varpi_{j}, \\
\left|\varphi_{j}\right| & \geq \frac{C_{1}}{N^{3 / 4}} \quad \text { on } \bar{\varpi}_{j}, \\
\left|\varphi_{j}\right| & \geq C_{1} N^{9 / 4} & \text { on } \omega_{j} .
\end{array}
$$

where $C_{1}$ is given in (1.11).

Proof. By the definitions (3.12), (3.22), (3.23) and (3.30), and noticing that $Q^{-1}$ and $A$ commute (cf. (3.17)), we have

$$
\begin{aligned}
\varphi_{j-1} & =e^{-\mathrm{i} \tau} P^{-1} A Q^{-1} \tilde{\psi}=e^{-\mathrm{i} \tau} P^{-1} Q^{-1} A \tilde{\psi}, \\
\varphi_{j} & =e^{-\mathrm{i} \tau} P^{-1} A Q^{-1} \hat{\psi}=e^{-\mathrm{i} \tau} P^{-1} Q^{-1} A \hat{\psi} .
\end{aligned}
$$

Then

$$
\left|\varphi_{j-1}\right|=|A \tilde{\psi}|, \quad\left|\varphi_{j}\right|=|A \hat{\psi}|, \quad|\tilde{\psi}|=\left|A^{-1} P \varphi_{j-1}\right|, \quad|\hat{\psi}|=\left|A^{-1} P \varphi_{j}\right|,
$$

hold because $P, Q \in \mathrm{SO}(3)$. By (1.4) and (3.11),

$$
\begin{aligned}
& \left|\varphi_{j}-\varphi_{j-1}\right|=|A(\hat{\psi}-\tilde{\psi})| \leq\|A\||\hat{\psi}-\tilde{\psi}| \leq \sqrt{2}|\hat{\psi}-\tilde{\psi}|, \\
& \left|\varphi_{j}-\varphi_{j-1}\right|=|A(\hat{\psi}-\tilde{\psi})| \geq \frac{1}{\left\|A^{-1}\right\|}|\hat{\psi}-\tilde{\psi}| \geq \frac{1}{\sqrt{2} N^{1 / 4}}|\hat{\psi}-\tilde{\psi}|
\end{aligned}
$$


hold. Here, by (3.24), (3.29) and (3.31), we have

$$
\begin{aligned}
|\hat{\psi}-\tilde{\psi}| & =\left|\frac{1}{2}\left(\left(1-\hat{g}^{2}\right) \hat{\eta}-\left(1-g^{2}\right) \eta, \mathrm{i}\left(1+\hat{g}^{2}\right) \hat{\eta}-\mathrm{i}\left(1+g^{2}\right) \eta\right)\right| \\
& =\frac{1}{2}\left|\left(\left(1-\frac{g^{2}}{h^{2}}\right) h \eta-\left(1-g^{2}\right) \eta, \mathrm{i}\left(1+\frac{g^{2}}{h^{2}}\right) h \eta-\mathrm{i}\left(1+g^{2}\right) \eta\right)\right| \\
& =\frac{1}{2}\left|(h-1)\left(\left(1+\frac{g^{2}}{h}\right), \mathrm{i}\left(1-\frac{g^{2}}{h}\right)\right) \eta\right| \\
& =\frac{1}{2}|h-1||\eta|\left(\left|1+\frac{g^{2}}{h}\right|^{2}+\left|1-\frac{g^{2}}{h}\right|^{2}\right)^{1 / 2} \leq \frac{1}{2}|h-1||\eta|\left(\left|1+\frac{g^{2}}{h}\right|+\left|1-\frac{g^{2}}{h}\right|\right) \\
& \leq|h-1||\eta|\left(1+\frac{|g|^{2}}{|h|}\right) \leq|h-1||\eta|\left(1+\frac{|g|^{2}}{1-|h-1|}\right) \\
& \leq|h-1| \frac{\left(1+|g|^{2}\right)|\eta|}{1-|h-1|}=\sqrt{2}|\tilde{\psi}| \frac{|h-1|}{1-|h-1|} .
\end{aligned}
$$

Since $h$ is taken as in Lemma 3.6 and $P \in \mathrm{SO}(3)$,

$$
\begin{array}{rlrl}
|\hat{\psi}-\tilde{\psi}| & \leq \sqrt{2}|\tilde{\psi}| \frac{\varepsilon_{1}}{1-\varepsilon_{1}}=\sqrt{2}|\tilde{\psi}| \frac{\varepsilon}{4 \sqrt{2} \mu_{j-1} N^{9 / 4}} & & \text { (Lemma 3.6, (3.2 })) \\
& =\left|A^{-1} P \varphi_{j-1}\right| \frac{\varepsilon}{4 \mu_{j-1} N^{9 / 4}} \leq|| A^{-1}||\left|P \varphi_{j-1}\right| \frac{\varepsilon}{4 \mu_{j-1} N^{9 / 4}} & & \text { (by }(3.37),(1.4)) \\
& \leq \sqrt{2} N^{1 / 4}\left|\varphi_{j-1}\right| \frac{\varepsilon}{4 \mu_{j-1} N^{9 / 4}} & & \text { (by (3.11)) } \\
& \leq \sqrt{2} N^{1 / 4} \mu_{j-1} \frac{\varepsilon}{4 \mu_{j-1} N^{9 / 4}}=\frac{\varepsilon}{2 \sqrt{2} N^{2}} & \text { (by (3.28)) }
\end{array}
$$

holds on $\overline{\mathbf{D}}_{1} \backslash \varpi_{j}$. Thus, by (3.38) $\left|\varphi_{j}-\varphi_{j-1}\right| \leq \sqrt{2}|\hat{\psi}-\tilde{\psi}| \leq \varepsilon /\left(2 N^{2}\right)$, which is (3.34).

Next, on $\bar{\varpi}_{j}$, it holds that

$$
\begin{array}{rlrl}
\left|\varphi_{j}\right| & =|A \hat{\psi}| \geq \frac{1}{\left\|A^{-1}\right\|}|\hat{\psi}| \geq \frac{1}{\sqrt{2} N^{1 / 4}}|\hat{\psi}| \quad(\text { by }(3.37),(1.4),(3.11)) \\
& =\frac{1}{\sqrt{2} N^{1 / 4}} \frac{1}{\sqrt{2}}\left(1+|\hat{g}|^{2}\right)|| \hat{\eta} \mid \\
& =\frac{1}{2 N^{1 / 4}}\left(1+|\hat{g}|^{2}\right)|\hat{\eta}| & \text { (by }(3.29),(1.9)) .
\end{array}
$$

Moreover 


$$
\begin{array}{rlr}
\left|\varphi_{j}\right| & \geq \frac{1}{N^{1 / 4}}|\hat{g} \hat{\eta}|=\frac{1}{N^{1 / 4}}|g \eta| & \text { (by (3.39), (3.31)) } \\
& =\frac{\sqrt{2}}{N^{1 / 4}} \frac{1}{\sqrt{2}}\left(1+|g|^{2}\right)|\eta| \frac{|g|}{1+|g|^{2}}=\frac{\sqrt{2}|\tilde{\psi}|}{N^{1 / 4}} \frac{|g|}{1+|g|^{2}} \quad \text { (by (3.24)) } \\
& =\frac{\sqrt{2}\left|A^{-1} P \varphi_{j-1}\right|}{N^{1 / 4}} \frac{|g|}{1+|g|^{2}} \geq \frac{\sqrt{2}\left|\varphi_{j-1}\right|}{N^{1 / 4}|| A \mid} \frac{|g|}{1+|g|^{2}} \quad(\text { by } \quad \text { (3.37), (1.4), } P \in \operatorname{SO}(3)) \\
& \geq \frac{\left|\varphi_{j-1}\right|}{N^{1 / 4}} \frac{|g|}{1+|g|^{2}} \geq \frac{\left|\varphi_{j-1}\right|}{N^{1 / 4}} \frac{2 \sqrt{N}}{1+4 N} \quad \text { (by (3.11), Lemma 3.4) } \\
& \geq \frac{v}{2 N^{1 / 4}} \frac{2 \sqrt{N}}{1+4 N}=\frac{v}{N^{3 / 4}} \frac{1}{4+1 / N} \geq \frac{v}{5 N^{3 / 4}}=\frac{C_{1}}{N^{3 / 4}} \quad \text { (Lemma 2.2, (1.11)). }
\end{array}
$$

Thus, we have (3.35).

Since $\omega_{j} \subset \varpi_{j}$, we have on $\omega_{j}$ that

$$
\begin{aligned}
& \left|\varphi_{j}\right| \geq \frac{1}{2 N^{1 / 4}}\left(1+|\hat{g}|^{2}\right)|\hat{\eta}| \geq \frac{1}{2 N^{1 / 4}}|\hat{\eta}| \\
& =\frac{1}{2 N^{1 / 4}}|h||\eta|=\frac{\sqrt{2}}{2 N^{1 / 4}} \frac{1}{\sqrt{2}}\left(1+|g|^{2}\right)|\eta| \frac{|h|}{1+|g|^{2}} \\
& =\frac{|h|}{\sqrt{2} N^{1 / 4}}|\tilde{\psi}| \frac{1}{1+|g|^{2}}=\frac{|h|}{\sqrt{2} N^{1 / 4}}\left|A^{-1} P \varphi_{j-1}\right| \frac{1}{1+|g|^{2}} \\
& \geq \frac{|h|}{\|A\| \sqrt{2} N^{1 / 4}}\left|P \varphi_{j-1}\right| \frac{1}{1+|g|^{2}} \geq \frac{|h|}{2 N^{1 / 4}}\left|P \varphi_{j-1}\right| \frac{1}{1+|g|^{2}} \\
& \geq \frac{|h|}{2 N^{1 / 4}}\left|\varphi_{j-1}\right| \frac{1}{1+4 N} \geq \frac{|h|}{2 N^{1 / 4}} \frac{v}{2} \frac{1}{1+4 N} \quad(P \in \operatorname{SO}(3), \text { Lemmas 3.4, 2.2) } \\
& \geq \frac{|h| v}{4 N^{1 / 4}} \frac{1}{5 N}=\frac{v}{20 N^{5 / 4}}|h| \geq \frac{v}{20 N^{5 / 4}}(T-|h-T|) \\
& \geq \frac{v}{20 N^{5 / 4}} 4 N^{7 / 2}=\frac{v}{5} N^{9 / 4}=C_{1} N^{9 / 4}
\end{aligned}
$$

Hence we have (3.36).

Thus we have $\left\{F_{j}\right\}$ and $\left\{\boldsymbol{v}_{j}\right\}$ satisfying properties (K-0)-(K-6) in Lemma 1.4.

\section{A proof of Proposition 1.3}

In this section, we prove Proposition 1.3. We take the sequences $\left\{F_{j}\right\}$ and $\left\{\boldsymbol{v}_{j}\right\}$ as in the Key Lemma 1.4, and set

$$
Y:=F_{2 N} .
$$


Recall that $X=F_{0}$ by $(\mathrm{K}-0)$. Then we shall prove $(\mathrm{Y}-1)-(\mathrm{Y}-3)$ in Proposition 1.3.

LEMMA 4.1. It holds that

$$
\left|\varphi_{Y}-\varphi_{X}\right| \leq \frac{\varepsilon}{N}, \quad \text { and } \quad|Y-X| \leq \frac{2 \varepsilon}{N} \quad \text { on } \quad \overline{\mathbf{D}}_{1} \backslash\left(\varpi_{1} \cup \cdots \cup \varpi_{2 N}\right),
$$

where $\varphi_{X}=d X / d z$ and $\varphi_{Y}=d Y / d z$.

Proof. By (K-2) of the Key Lemma 1.4,

$$
\left|\varphi_{Y}-\varphi_{X}\right|=\left|\varphi_{2 N}-\varphi_{0}\right| \leq\left|\varphi_{2 N}-\varphi_{2 N-1}\right|+\cdots+\left|\varphi_{1}-\varphi_{0}\right| \leq 2 N \cdot \frac{\varepsilon}{2 N^{2}}=\frac{\varepsilon}{N}
$$

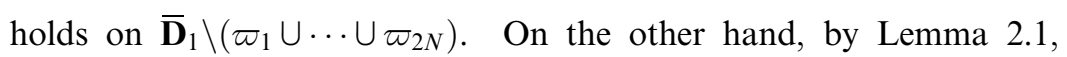

$$
|Y-X|=\left|F_{2 N}-F_{0}\right| \leq\left|F_{2 N}-F_{2 N-1}\right|+\cdots+\left|F_{1}-F_{0}\right| \leq 2 N \cdot \frac{\varepsilon}{N^{2}}=\frac{2 \varepsilon}{N}
$$

holds on $\overline{\mathbf{D}}_{1} \backslash\left(\varpi_{1} \cup \cdots \cup \varpi_{2 N}\right)$.

COROllary 4.2 (the conclusion (Y-1)). It holds that

$$
\left|\varphi_{Y}-\varphi_{X}\right|<\varepsilon \text { and }|Y-X|<\varepsilon \text { on } \mathbf{D}_{1-\varepsilon} .
$$

Proof. Note that we take the labyrinth as in Appendix A. Here, by (1.14),

$$
\frac{2}{N}+\frac{1}{8 N^{3}}=\frac{1}{N}\left(2+\frac{1}{8 N^{2}}\right)<\frac{3}{N} \leq \varepsilon
$$

holds. Then by (2) of Lemma A.1 in Appendix A, we have that

$$
\mathbf{D}_{1-\varepsilon} \subset \overline{\mathbf{D}}_{1} \backslash\left(\varpi_{1} \cup \cdots \cup \varpi_{2 N}\right) .
$$

Thus, by Lemma 4.1 and (1.13), it holds on $\mathbf{D}_{1-\varepsilon}$ that

$$
\left|\varphi_{Y}-\varphi_{X}\right|=\left|\varphi_{2 N}-\varphi_{0}\right| \leq \frac{\varepsilon}{N}<\varepsilon, \quad|Y-X|=\left|F_{2 N}-F_{0}\right| \leq \frac{2 \varepsilon}{N}<\varepsilon
$$

LEMma 4.3. The function $\varphi_{Y}=\varphi_{2 N}$ satisfies

$$
\left|\varphi_{Y}\right| \geq \begin{cases}\frac{C_{1}}{2} N^{9 / 4} & \text { on } \omega_{1} \cup \cdots \cup \omega_{2 N} \\ \frac{C_{1}}{2 N^{3 / 4}} & \text { on } \overline{\mathbf{D}}_{1}\end{cases}
$$


Proof. On $\omega_{j}$,

$$
\begin{aligned}
\left|\varphi_{Y}\right| & =\left|\varphi_{2 N}\right| \geq\left|\varphi_{j}\right|-\left|\varphi_{2 N}-\varphi_{2 N-1}\right|-\cdots-\left|\varphi_{j+1}-\varphi_{j}\right| \\
& \geq C_{1} N^{9 / 4}-\frac{(2 N-j+1) \varepsilon}{2 N^{2}} \quad(\text { by }(\mathrm{K}-3),(\mathrm{K}-2)) \\
& \geq C_{1} N^{9 / 4}-\frac{\varepsilon}{N}=N^{9 / 4}\left(C_{1}-\frac{\varepsilon}{N^{13 / 4}}\right) \\
& \geq N^{9 / 4}\left(C_{1}-\frac{\varepsilon}{N^{1 / 4}}\right) \geq \frac{C_{1}}{2} N^{9 / 4} \quad(\text { by }(1.14)) .
\end{aligned}
$$

On the other hand, on $\varpi_{j}$, we have

$$
\begin{aligned}
\left|\varphi_{Y}\right| & =\left|\varphi_{2 N}\right| \geq\left|\varphi_{j}\right|-\left|\varphi_{2 N}-\varphi_{2 N-1}\right|-\cdots-\left|\varphi_{j+1}-\varphi_{j}\right| \\
& \geq \frac{C_{1}}{N^{3 / 4}}-\frac{(2 N-j+1) \varepsilon}{2 N^{2}} \quad(\text { by }(\mathrm{K}-3),(\mathrm{K}-2)) \\
& \geq \frac{C_{1}}{N^{3 / 4}}-\frac{\varepsilon}{N}=\frac{1}{N^{3 / 4}}\left(C_{1}-\frac{\varepsilon}{N^{1 / 4}}\right) \geq \frac{C_{1}}{2 N^{3 / 4}} \quad(\text { by }(1.14)) .
\end{aligned}
$$

Finally, on $\overline{\mathbf{D}}_{1} \backslash\left(\varpi_{1} \cup \cdots \cup \varpi_{2 N}\right)$,

$$
\begin{aligned}
\left|\varphi_{Y}\right| & =\left|\varphi_{2 N}\right| \geq\left|\varphi_{0}\right|-\left|\varphi_{2 N}-\varphi_{2 N-1}\right|-\cdots-\left|\varphi_{1}-\varphi_{0}\right| \\
& \geq\left|\varphi_{0}\right|-2 N \cdot \frac{\varepsilon}{2 N^{2}} \geq v-\frac{\varepsilon}{N} \quad(\text { by }(\mathrm{K}-2),(1.16)) \\
& \geq v-\frac{\varepsilon}{N^{3 / 4}} \geq \frac{C_{1}}{2 N^{3 / 4}} \quad(\text { by }(1.14)) .
\end{aligned}
$$

Hence we have the conclusion.

Corollary 4.4 (the conclusion $(\mathrm{Y}-2)$ ). The disc $\left(\mathbf{D}_{1}, d s_{Y}^{2}\right)$ contains a geodesic disc $\mathscr{D}$ centered at 0 with radius $\rho+s$.

Proof. The induced metric $d s_{Y}^{2}$ is expressed as

$$
d s_{Y}^{2}=\left|\varphi_{Y}\right|^{2}|d z|^{2} .
$$

Consider a Riemannian metric

$$
d s^{2}:=\left(\frac{2 N^{3 / 4}}{C_{1}}\right)^{2} d s_{Y}^{2}=\lambda^{2}|d z|^{2}, \quad\left(\lambda:=\frac{2 N^{3 / 4}}{C_{1}}\left|\varphi_{Y}\right|\right) .
$$

Then by Lemma 4.3, $d s^{2}$ satisfies the assumptions of Lemma A.4 in Appendix A. Thus, we have

$$
\operatorname{dist}_{d s^{2}}\left(0, \partial \overline{\mathbf{D}}_{1}\right) \geq N
$$


where dist $d s^{2}$ denotes the distance function with respect to $d s^{2}$. Then by (1.14), we have

$$
\operatorname{dist}_{d s_{Y}^{2}}\left(0, \partial \overline{\mathbf{D}}_{1}\right) \geq \frac{C_{1}}{2 N^{3 / 4}} N=\frac{C_{1} N^{1 / 4}}{2} \geq \rho+s .
$$

Hence we have the conclusion.

By Corollary 4.4, one can take a geodesic disc $\mathscr{D}$ of $\left(\mathbf{D}_{1}, d s_{Y}^{2}\right)$ centered at the origin with radius $\rho+s$. We fix $q \in \partial \mathscr{D}$, and will prove (Y-3) of Proposition 1.3 from now on:

First, we assume $q \in \varpi_{j}$ for some $j \in\{1, \ldots, 2 N\}$ (otherwise, the proof of (Y-3) is rather easy). Since $q \in \partial \mathscr{D}$, there exists a $d s_{Y}^{2}$-geodesic $\gamma$ joining 0 and $q$ with length $\rho+s$. Since $d s_{Y}^{2}$ is a Riemannian metric of non-positive Gaussian curvature,

an arbitrary subarc of $\gamma$ is the shortest geodesic.

Hence the image of $\gamma$ is contained in $\mathscr{D}$.

LEMma 4.5. The Euclidean length of $\gamma$ satisfies

$$
\text { Length }_{\mathbf{C}}(\gamma) \leq \frac{2(\rho+s)}{C_{1}} N^{3 / 4}
$$

Proof. Since the $d s_{Y}^{2}$-arclength of $\gamma$ is $\rho+s$, Lemma 4.3 implies that

$$
\rho+s=\int_{\gamma}\left|\varphi_{Y}\right||d z| \geq \int_{\gamma} \frac{C_{1}}{2 N^{3 / 4}}|d z|=\frac{C_{1}}{2 N^{3 / 4}} \operatorname{Length}_{\mathbf{C}}(\gamma) .
$$

Hence we have the conclusion.

Now, take points $\check{q}, \tilde{q} \in \mathscr{D}$ on the arc $\gamma$ such that

- $\breve{q} \in \partial \varpi_{j}$ and the subarc of $\gamma$ joining $\check{q}$ and $q$ is contained in $\bar{\varpi}_{j}$, namely, $\check{q}$ is the final point where $\gamma$ meets $\partial \varpi_{j}$,

- and the subarc of $\gamma$ joining 0 and $\tilde{q} \in \partial \mathbf{D}_{1-\frac{2}{N}-\frac{1}{8 N^{3}}}$ contained in $\overline{\mathbf{D}}_{1-\frac{2}{N}-\frac{1}{8 N^{3}}}$; namely, $\tilde{q}$ is the first point where $\gamma$ meets $\partial \mathbf{D}_{1-\frac{2}{N}-\frac{1}{8 N^{3}}}$.

See Figure 1.

LEMMA 4.6. It holds that

$$
\begin{aligned}
& \left|F_{l}(\check{q})\right| \leq r+\frac{2 \varepsilon}{N} \quad(l=0, \ldots, 2 N), \\
& \left|F_{j-1}(q)\right| \leq r+\frac{2 \varepsilon}{N}, \\
& \left|F_{2 N}(q)-F_{j}(q)\right| \leq \frac{2 \varepsilon}{N}, \quad\left|F_{2 N}(\check{q})-F_{j}(\check{q})\right| \leq \frac{2 \varepsilon}{N}, \\
& \left|F_{2 N}(q)-F_{2 N}(\check{q})\right| \leq s+\frac{c_{2}}{N^{1 / 4}},
\end{aligned}
$$

where $c_{2}$ is defined by (1.12). 


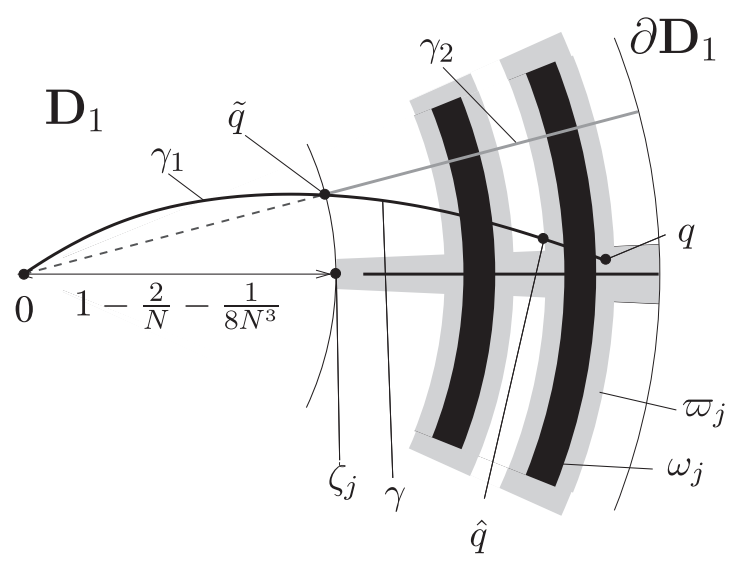

FIGURE 1. The curve $\gamma$ and points $\check{q}, \tilde{q}$

Proof. Since $\check{q} \notin \varpi_{1} \cup \cdots \cup \varpi_{2 N}$, Lemma 2.1 and the assumption (X-3) of the Proposition 1.3 imply

$$
\begin{aligned}
\left|F_{l}(\check{q})\right| & \leq\left|F_{0}(\check{q})\right|+\left|F_{1}(\check{q})-F_{0}(\check{q})\right|+\cdots+\left|F_{l}(\check{q})-F_{l-1}(\check{q})\right| \\
& \leq r+\frac{l \varepsilon}{N^{2}} \leq r+\frac{2 \varepsilon}{N} .
\end{aligned}
$$

Hence we have (4.4). A similar reasoning proves (4.5).

If $j=2 N$, (4.6) is obvious. When $j \leq 2 N-1$, since $q \notin \varpi_{j+1} \cup \cdots \cup \varpi_{2 N}$, Lemma 2.1 implies

$$
\begin{aligned}
\left|F_{2 N}(q)-F_{j}(q)\right| & \leq\left|F_{2 N}(q)-F_{2 N-1}(q)\right|+\cdots+\left|F_{j+1}(q)-F_{j}(q)\right| \\
& \leq \frac{(2 N-j) \varepsilon}{N^{2}}<\frac{2 \varepsilon}{N} .
\end{aligned}
$$

Then the first inequality of (4.6) holds. Similarly, we have the second inequality of (4.6).

Let $\gamma_{1}$ be the subarc of the geodesic $\gamma$ joining 0 and $\tilde{q}$, and let $\gamma_{2}$ be the line segment joining $\tilde{q}$ and $\partial \mathbf{D}_{1}$ which is contained in the line $\{t \tilde{q} \mid t \in \mathbf{R}\}$, see Figure 1. Since $\gamma_{1} \cup \gamma_{2}$ is a path joining 0 and $\partial \mathbf{D}_{1}$, the assumption (X-2) and (K-0) imply that

$$
\operatorname{Length}_{d s_{X}^{2}}\left(\gamma_{1} \cup \gamma_{2}\right)=\int_{\gamma_{1} \cup \gamma_{2}}\left|\varphi_{X}(z)\right||d z| \geq \operatorname{dist}_{d s_{X}^{2}}\left(0, \partial \mathbf{D}_{1}\right) \geq \rho,
$$

where Length ${ }_{d s_{X}^{2}}\left(\gamma_{1} \cup \gamma_{2}\right)$ is the length of the curve $\gamma_{1} \cup \gamma_{2}$ with respect to the metric $d s_{X}^{2}$. On the other hand, by (1.16), we have 


$$
\begin{aligned}
\operatorname{Length}_{d s_{X}^{2}}\left(\gamma_{2}\right) & =\int_{\gamma_{2}}\left|\varphi_{X}(z)\right||d z| \leq \mu \cdot \operatorname{Length}_{\mathbf{C}}\left(\gamma_{2}\right) \\
& =\mu\left(\frac{2}{N}+\frac{1}{8 N^{3}}\right)=\frac{\mu}{N}\left(2+\frac{1}{8 N^{2}}\right) \leq \frac{3 \mu}{N} .
\end{aligned}
$$

Hence we have

$$
\int_{\gamma_{1}}\left|\varphi_{X}(z)\right||d z|=\int_{\gamma_{1} \cup \gamma_{2}}\left|\varphi_{X}(z)\right||d z|-\int_{\gamma_{2}}\left|\varphi_{X}(z)\right||d z| \geq \rho-\frac{3 \mu}{N} .
$$

Since $\gamma_{1}$ is contained in the subarc of $\gamma$ joining 0 and $\check{q}$, and taking into account that $Y=F_{2 N}$ (cf. (4.1)), we have

$$
\begin{aligned}
& \operatorname{dist}_{d s_{Y}^{2}}(0, \check{q}) \geq \operatorname{dist}_{d s_{Y}^{2}}(0, \tilde{q})=\int_{\gamma_{1}}\left|\varphi_{Y}(z)\right||d z| \\
& =\int_{\gamma_{1}}\left(\left|\varphi_{Y}(z)\right|-\left|\varphi_{X}(z)\right|\right)|d z|+\int_{\gamma_{1}}\left|\varphi_{X}(z)\right||d z| \\
& \geq-\int_{\gamma_{1}}\left|\varphi_{Y}(z)-\varphi_{X}(z)\right||d z|+\int_{\gamma_{1}}\left|\varphi_{X}(z)\right||d z| \\
& \geq-\int_{\gamma_{1}}\left|\varphi_{Y}(z)-\varphi_{X}(z)\right||d z|+\rho-\frac{3 \mu}{N} \\
& \geq-\int_{\gamma_{1}} \frac{\varepsilon}{N}|d z|+\rho-\frac{3 \mu}{N} \\
& \geq- \text { Length }_{\mathbf{C}}(\gamma) \frac{\varepsilon}{N}+\rho-\frac{3 \mu}{N} \\
& \geq-\frac{2 \varepsilon(\rho+s)}{C_{1} N^{1 / 4}}+\rho-\frac{3 \mu}{N} \geq-\frac{2 \varepsilon(\rho+s)}{C_{1} N^{1 / 4}}+\rho-\frac{3 \mu}{N^{1 / 4}} \\
& =\rho-\frac{1}{N^{1 / 4}}\left(3 \mu+\frac{2 \varepsilon(\rho+s)}{C_{1}}\right)=\rho-\frac{c_{2}}{N^{1 / 4}} \\
& \left(\gamma_{1} \subset \gamma\right)
\end{aligned}
$$

Here, since $\check{q}$ lies on the geodesic $\gamma$ joining 0 and $q$, (4.3) implies

$$
\begin{aligned}
\left|F_{2 N}(q)-F_{2 N}(\check{q})\right| & \leq \operatorname{dist}_{d s_{Y}^{2}}(q, \check{q})=\operatorname{dist}_{d s_{Y}^{2}}(0, q)-\operatorname{dist}_{d s_{Y}^{2}}(0, \check{q}) \\
& =\rho+s-\operatorname{dist}_{d s_{Y}^{2}}(0, \check{q}) \\
& \leq \rho+s-\left(\rho-\frac{c_{2}}{N^{1 / 4}}\right)=s+\frac{c_{2}}{N^{1 / 4}} .
\end{aligned}
$$

Thus (4.7) is obtained.

We first consider the case that $q \in \varpi_{j}$ and $\left|F_{j-1}\left(\zeta_{j}\right)\right|>1 / \sqrt{N}$. 
LEMMA 4.7. When $q \in \varpi_{j}$ and $\left|F_{j-1}\left(\zeta_{j}\right)\right|>1 / \sqrt{N}$,

$$
\left|F_{j}(q)\right| \leq \sqrt{r^{2}+s^{2}}+\frac{c_{3}}{N^{1 / 4}}
$$

holds.

Proof. Let $\boldsymbol{v}_{j} \in \mathbf{C}^{3}$ be the unit vector as in (K-4)-(K-6), and denote $\left(\boldsymbol{v}_{j}\right)^{\perp}:=\left(\right.$ the orthogonal complement of $\boldsymbol{v}_{j}$ with respect to $\left.\langle\rangle,\right)$.

Then $\left(\boldsymbol{v}_{j}\right)^{\perp}$ is a (complex) 2-dimensional subspace of $\mathbf{C}^{3}$. Denote by $\Pi_{j}$ the orthogonal projection

$$
\Pi_{j}: \mathbf{C}^{3} \ni \boldsymbol{x} \mapsto \boldsymbol{x}-\left\langle\boldsymbol{x}, \boldsymbol{v}_{j}\right\rangle \boldsymbol{v}_{j} \in\left(\boldsymbol{v}_{j}\right)^{\perp}
$$

with respect to the Hermitian inner product $\langle$,$\rangle . Then for any vector \boldsymbol{x} \in \mathbf{C}^{3}$,

$$
|\boldsymbol{x}|^{2}=\left|\left\langle\boldsymbol{x}, \boldsymbol{v}_{j}\right\rangle\right|^{2}+\left|\Pi_{j} \boldsymbol{x}\right|^{2}
$$

holds. Thus, we have

$$
\begin{aligned}
\left|\Pi_{j} F_{j}(q)\right| \leq & \left|\Pi_{j} F_{j}(q)-\Pi_{j} F_{j}(\check{q})\right|+\left|\Pi_{j} F_{j}(\check{q})\right| \\
\leq & \left|\Pi_{j}\left(F_{j}(q)-F_{j}(\check{q})\right)\right|+\left|\Pi_{j} F_{j}(\check{q})\right| \\
\leq & \left|F_{j}(q)-F_{j}(\check{q})\right|+\left|\Pi_{j} F_{j}(\check{q})\right| \\
\leq & \left|F_{j}(q)-F_{j}(\check{q})\right|+\left|\Pi_{j}\left(F_{j}(\check{q})-F_{j-1}(\check{q})\right)\right|+\left|\Pi_{j} F_{j-1}(\check{q})\right| \\
\leq & \left|F_{j}(q)-F_{j}(\check{q})\right|+\left|F_{j}(\check{q})-F_{j-1}(\check{q})\right|+\left|\Pi_{j} F_{j-1}(\check{q})\right| \quad(\text { by } \quad(4.12)) \\
\leq & \left|F_{2 N}(q)-F_{2 N}(\check{q})\right|+\left|F_{2 N}(q)-F_{j}(q)\right|+\left|F_{2 N}(\check{q})-F_{j}(\check{q})\right| \\
& +\left|F_{j}(\check{q})-F_{j-1}(\check{q})\right|+\left|\Pi_{j} F_{j-1}(\check{q})\right| \\
\leq & \left(s+\frac{c_{2}}{N^{1 / 4}}\right)+\frac{2 \varepsilon}{N}+\frac{2 \varepsilon}{N} \\
& +\left|F_{j}(\check{q})-F_{j-1}(\check{q})\right|+\left|\Pi_{j} F_{j-1}(\check{q})\right| \\
\leq & \left(s+\frac{c_{2}}{N^{1 / 4}}\right)+\frac{4 \varepsilon}{N}+\frac{\varepsilon}{N^{2}}+\left|\Pi_{j} F_{j-1}(\check{q})\right| \\
\leq & s+\frac{1}{N^{1 / 4}}\left(c_{2}+\frac{4 \varepsilon}{N^{3 / 4}}+\frac{\varepsilon}{N^{7 / 4}}\right)+\left|\Pi_{j} F_{j-1}(\check{q})\right| \\
\leq & s+\frac{c_{2}+5 \varepsilon}{N^{1 / 4}}+\left|\Pi_{j} F_{j-1}(\check{q})\right| .
\end{aligned}
$$

Hence we have

$$
\left|\Pi_{j} F_{j}(q)\right| \leq s+\frac{c_{2}+5 \varepsilon}{N^{1 / 4}}+\left|\Pi_{j} F_{j-1}(\check{q})\right|
$$


Here, we assume $F_{j-1}(\check{q}) \neq 0$. Since $\check{q} \in \bar{\varpi}_{j}$, we have

$$
\begin{aligned}
\left|\Pi_{j} F_{j-1}(\check{q})\right| & \left.=\sqrt{\left|F_{j-1}(\check{q})\right|^{2}-\left|\left(F_{j-1}(\check{q}), \boldsymbol{v}_{j}\right)\right|^{2}} \quad \text { (by }(4.12)\right) \\
& \left.=\left|F_{j-1}(\check{q})\right| \sqrt{1-\left|\left\langle\frac{F_{j-1}(\check{q})}{\left|F_{j-1}(\check{q})\right|}, \boldsymbol{v}_{j}\right\rangle\right|^{2}} \quad \quad \text { by }(\mathrm{K}-5)\right) \\
& \leq\left|F_{j-1}(\check{q})\right| \sqrt{1-\left(1-\frac{C_{2}}{\sqrt{N}}\right)^{2}} \quad \text { (by (4.4) in Lemma 4.6) } \\
& =\left|F_{j-1}(\check{q})\right| \sqrt{\frac{2 C_{2}}{\sqrt{N}}-\frac{\left(C_{2}\right)^{2}}{N} \leq\left|F_{j-1}(\check{q})\right|} \sqrt{\frac{2 C_{2}}{\sqrt{N}}} \\
& =\left|F_{j-1}(\check{q})\right| \frac{\sqrt{2 C_{2}}}{N^{1 / 4}} \leq\left(r+\frac{2 \varepsilon}{N}\right) \cdot \frac{\sqrt{2 C_{2}}}{N^{1 / 4}} \quad \text { in } \quad \\
& \leq \frac{(r+2 \varepsilon) \sqrt{2 C_{2}}}{N^{1 / 4}} .
\end{aligned}
$$

Then by (4.13), we have

$$
\left|\Pi_{j} F_{j}(q)\right| \leq s+\frac{\alpha}{N^{1 / 4}} \quad\left(\alpha:=c_{2}+5 \varepsilon+(r+2 \varepsilon) \sqrt{2 C_{2}}\right)
$$

when $F_{j-1}(\check{q}) \neq 0$. Otherwise, namely when $F_{j-1}(\check{q})=0$, (4.14) holds trivially. Thus,

$$
\begin{array}{rlrl}
\left|F_{j}(q)\right| & =\sqrt{\left|\left\langle F_{j}(q), \boldsymbol{v}_{j}\right\rangle\right|^{2}+\left|\Pi_{j} F_{j}(q)\right|^{2}} & & (\text { by }(4.12)) \\
& =\sqrt{\left|\left\langle F_{j-1}(q), \boldsymbol{v}_{j}\right\rangle\right|^{2}+\left|\Pi_{j} F_{j}(q)\right|^{2}} & & (\text { by }(\mathrm{K}-6)) \\
& \leq \sqrt{\left|F_{j-1}(q)\right|^{2}+\left|\Pi_{j} F_{j}(q)\right|^{2}} & \\
& \leq \sqrt{\left(r+\frac{2 \varepsilon}{N}\right)^{2}+\left(s+\frac{\alpha}{N^{1 / 4}}\right)^{2}}=\sqrt{\left(r^{2}+s^{2}\right)+\frac{2}{N^{1 / 4}} \beta,} \quad & \text { (by }(4.5),(4.14)),
\end{array}
$$

where

$$
\beta:=s \alpha+\left(\frac{\alpha^{2}}{2 N^{1 / 4}}+\frac{2 r \varepsilon}{N^{3 / 4}}+\frac{2 \varepsilon^{2}}{N^{7 / 4}}\right) \leq s \alpha+\frac{\alpha^{2}}{2}+2 r \varepsilon+2 \varepsilon^{2}=c_{3} \sqrt{r^{2}+s^{2}}
$$

and $c_{3}$ is the constant as in (1.12). Hence by the inequality $\sqrt{1+x} \leq 1+(x / 2)$, 


$$
\begin{aligned}
\left|F_{j}(q)\right| & \leq \sqrt{r^{2}+s^{2}} \sqrt{1+\frac{2}{N^{1 / 4}} \frac{\beta}{r^{2}+s^{2}}} \leq \sqrt{r^{2}+s^{2}}\left(1+\frac{1}{N^{1 / 4}} \frac{\beta}{r^{2}+s^{2}}\right) \\
& \leq \sqrt{r^{2}+s^{2}}\left(1+\frac{1}{N^{1 / 4}} \frac{c_{3}}{\sqrt{r^{2}+s^{2}}}\right) \leq \sqrt{r^{2}+s^{2}}+\frac{c_{3}}{N^{1 / 4}}
\end{aligned}
$$

holds, which is the conclusion.

COROLlary 4.8. Under the assumption of Lemma 4.7, we have

$$
|Y(q)|=\left|F_{2 N}(q)\right| \leq \sqrt{r^{2}+s^{2}}+\varepsilon \quad \text { for } q \in\left(\partial \mathscr{D} \cap \varpi_{j}\right) .
$$

Proof.

$$
\begin{array}{rlrl}
\left|F_{2 N}(q)\right| & \leq\left|F_{j}(q)\right|+\left|F_{2 N}(q)-F_{j}(q)\right| \leq\left|F_{j}(q)\right|+\frac{2 \varepsilon}{N} & & (\text { by }(4.6) \text { in Lemma 4.6) } \\
& \leq \sqrt{r^{2}+s^{2}}+\frac{c_{3}}{N^{1 / 4}}+\frac{2 \varepsilon}{N} & & (\text { Lemma 4.7) } \\
& =\sqrt{r^{2}+s^{2}}+\frac{1}{N^{1 / 4}}\left(c_{3}+\frac{2 \varepsilon}{N^{3 / 4}}\right) \\
& \leq \sqrt{r^{2}+s^{2}}+\frac{1}{N^{1 / 4}}\left(c_{3}+2 \varepsilon\right) \leq \sqrt{r^{2}+s^{2}}+\varepsilon \quad(\text { by }(1.15)) .
\end{array}
$$

Next we consider the case $q \in \varpi_{j}$ and $\left|F_{j-1}\left(\zeta_{j}\right)\right| \leq 1 / \sqrt{N}$.

LEMMA 4.9. When $\left|F_{j-1}\left(\zeta_{j}\right)\right| \leq 1 / \sqrt{N}$ and $q \in\left(\partial \mathscr{D} \cap \varpi_{j}\right)$,

$$
|Y(q)|=\left|F_{2 N}(q)\right| \leq \sqrt{r^{2}+s^{2}}+\varepsilon
$$

holds.

Proof. Since $\check{q} \in \partial \varpi_{j}$,

$$
\begin{aligned}
\left|F_{2 N}(q)\right| & \leq\left|F_{2 N}(q)-F_{2 N}(\check{q})\right|+\left|F_{2 N}(\check{q})\right| \leq\left(s+\frac{c_{2}}{N^{1 / 4}}\right)+\left|F_{2 N}(\check{q})\right| \\
& \leq\left(s+\frac{c_{2}}{N^{1 / 4}}\right)+\left|F_{2 N}(\check{q})-F_{j}(\check{q})\right|+\left|F_{j}(\check{q})\right| \\
& \leq\left(s+\frac{c_{2}}{N^{1 / 4}}\right)+\frac{2 \varepsilon}{N}+\left|F_{j}(\check{q})\right| \\
& \leq s+\frac{1}{N^{1 / 4}}\left(c_{2}+\frac{2 \varepsilon}{N^{3 / 4}}\right)+\left|F_{j}(\check{q})-F_{j-1}(\check{q})\right|+\left|F_{j-1}(\check{q})\right| \\
& \leq s+\frac{1}{N^{1 / 4}}\left(c_{2}+\frac{2 \varepsilon}{N^{3 / 4}}\right)+\frac{\varepsilon}{N^{2}}+\left|F_{j-1}(\check{q})\right|
\end{aligned}
$$




$$
\begin{aligned}
& \leq s+\frac{1}{N^{1 / 4}}\left(c_{2}+\frac{2 \varepsilon}{N^{3 / 4}}+\frac{\varepsilon}{N^{7 / 4}}\right)+\left|F_{j-1}(\check{q})-F_{j-1}\left(\zeta_{j}\right)\right|+\left|F_{j-1}\left(\zeta_{j}\right)\right| \\
& \leq s+\frac{1}{N^{1 / 4}}\left(c_{2}+\frac{2 \varepsilon}{N^{3 / 4}}+\frac{\varepsilon}{N^{7 / 4}}+\frac{6 \mu}{N^{3 / 4}}\right)+\left|F_{j-1}\left(\zeta_{j}\right)\right| \\
& \leq s+\frac{1}{N^{1 / 4}}\left(c_{2}+\frac{2 \varepsilon}{N^{3 / 4}}+\frac{\varepsilon}{N^{7 / 4}}+\frac{6 \mu}{N^{3 / 4}}+\frac{1}{N^{1 / 4}}\right) \\
& =s+\frac{1+c_{2}+6 \mu+3 \varepsilon}{N^{1 / 4}} \leq s+\varepsilon \leq \sqrt{r^{2}+s^{2}}+\varepsilon
\end{aligned}
$$

Thus we have the conclusion.

The remaining case is when

$$
q \in \partial \mathscr{D} \cap\left(\overline{\mathbf{D}}_{1} \backslash\left(\varpi_{1} \cup \cdots \cup \varpi_{2 N}\right)\right) .
$$

LEMMA 4.10. If $q$ satisfies (4.15), then

$$
\left|F_{2 N}(q)\right| \leq \sqrt{r^{2}+s^{2}}+\varepsilon
$$

holds.

Proof. By the assumption (X-3), $|X(q)|=\left|F_{0}(q)\right| \leq r$ holds. Then by Lemma 4.1 and (1.13), we have

$$
\left|F_{2 N}(q)\right| \leq\left|F_{0}(q)\right|+\left|F_{2 N}(q)-F_{0}(q)\right| \leq r+\frac{2 \varepsilon}{N} \leq r+\varepsilon \leq \sqrt{r^{2}+s^{2}}+\varepsilon .
$$

Summing up, Corollary 4.8 and Lemmas 4.9 and 4.10 imply (Y-3) of the Proposition 1.3.

\section{Appendix A. Labyrinth}

For the sake of completeness, we recall Nadirashvili's labyrinth (for further details we refer the reader to [7] or [4]).

For each number $k=0,1,2, \ldots, 2 N^{2}$, we set

$$
r_{k}:=1-\frac{k}{N^{3}} \quad\left(r_{0}=1, r_{1}=1-\frac{1}{N^{3}}, \ldots, r_{2 N^{2}}=1-\frac{2}{N}\right),
$$

and take a sequence of domains

$$
\mathbf{D}_{r_{k}}=\left\{z \in \mathbf{C} ;|z|<r_{k}\right\} \quad\left(k=0, \ldots, 2 N^{2}\right) .
$$

Since $\left\{r_{k}\right\}$ is decreasing in $k$, it holds that

$$
\mathbf{D}_{1}=\mathbf{D}_{r_{0}} \supset \mathbf{D}_{r_{1}} \supset \cdots \supset \mathbf{D}_{r_{2 N^{2}}}=\mathbf{D}_{1-\frac{2}{N}} .
$$


We denote the boundaries of $\mathbf{D}_{r_{k}}$ by

$$
S_{r_{k}}=\partial \mathbf{D}_{r_{k}}=\left\{z \in \mathbf{C} ;|z|=r_{k}\right\} .
$$

We set

$$
\mathscr{A}:=\overline{\mathbf{D}}_{1} \backslash \mathbf{D}_{r_{2 N 2}}=\overline{\mathbf{D}}_{1} \backslash \mathbf{D}_{1-\frac{2}{N}}
$$

and

$$
\begin{aligned}
& A:=\bigcup_{k=0}^{N^{2}-1}\left(\mathbf{D}_{r_{2 k}} \backslash \mathbf{D}_{r_{2 k+1}}\right)=\left(\mathbf{D}_{r_{0}} \backslash \mathbf{D}_{r_{1}}\right) \cup\left(\mathbf{D}_{r_{2}} \backslash \mathbf{D}_{r_{3}}\right) \cup \cdots \cup\left(\mathbf{D}_{r_{2 N^{2}-2}} \backslash \mathbf{D}_{r_{2 N^{2}-1}}\right), \\
& \tilde{A}:=\bigcup_{k=0}^{N^{2}-1}\left(\mathbf{D}_{r_{2 k+1}} \backslash \mathbf{D}_{r_{2 k+2}}\right)=\left(\mathbf{D}_{r_{1}} \backslash \mathbf{D}_{r_{2}}\right) \cup\left(\mathbf{D}_{r_{3}} \backslash \mathbf{D}_{r_{4}}\right) \cup \cdots \cup\left(\mathbf{D}_{r_{2 N^{2}-1}} \backslash \mathbf{D}_{r_{2 N^{2}}}\right) .
\end{aligned}
$$

Next, let

$$
L:=\left(\bigcup_{j=0}^{N-1} l_{2 j \pi / N}\right) \cap A, \quad \tilde{L}:=\left(\bigcup_{j=0}^{N-1} l_{(2 j+1) \pi / N}\right) \cap \tilde{A},
$$

where $l_{t}:=\left\{r e^{\mathrm{i} t} ; r \geq 0\right\}$, and set

$$
H:=L \cup \tilde{L} \cup S \quad\left(S:=\bigcup_{k=0}^{2 N^{2}} \partial \mathbf{D}_{r_{k}}=\bigcup_{k=0}^{2 N^{2}} S_{r_{k}}\right)
$$

We define

$$
\Omega:=\mathscr{A} \backslash U\left[\frac{1}{4 N^{3}}\right](H),
$$

where $U[\varepsilon](B)$ denotes the $\varepsilon$-neighborhood of the subset $B \subset \mathbf{C}$ (in the Euclidean distance). Note that each connected component of $\Omega$ has the width $1 /\left(2 N^{3}\right)$.

For each number $j=1, \ldots, 2 N$, we set

$$
\omega_{j}:=\left(l_{j \pi / N} \cap \mathscr{A}\right)
$$

$\cup\left(\right.$ the connected components of $\Omega$ intersecting with $\left.l_{j \pi / N}\right)$,

$$
\varpi_{j}:=U\left[\frac{1}{8 N^{3}}\right]\left(\omega_{j}\right)=\left(\text { the } \frac{1}{8 N^{3}} \text {-neighborhood of } \omega_{j}\right),
$$

$\bar{\varpi}_{j}:=$ the closure of $\varpi_{j}$.

Finally we denote by $\zeta_{j}$ the "base point" of $\varpi_{j}$ :

$$
\zeta_{j}:=\left(1-\frac{2}{N}-\frac{1}{8 N^{3}}\right) e^{\mathrm{i} \pi j / N} \in \partial \varpi_{j} \quad(j=1, \ldots, 2 N)
$$

(see Figure 3). 

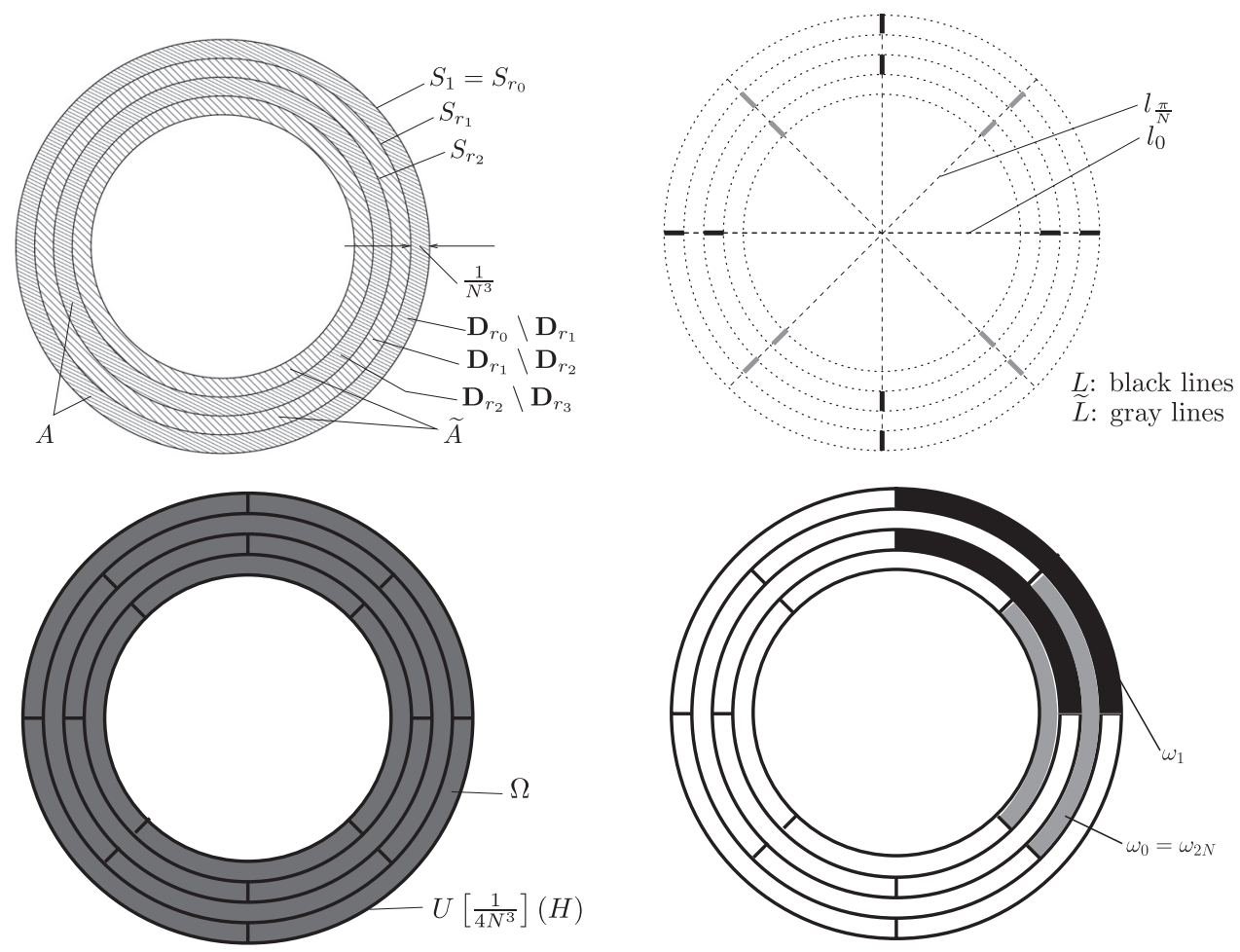

FIGURE 2. The labyrinth

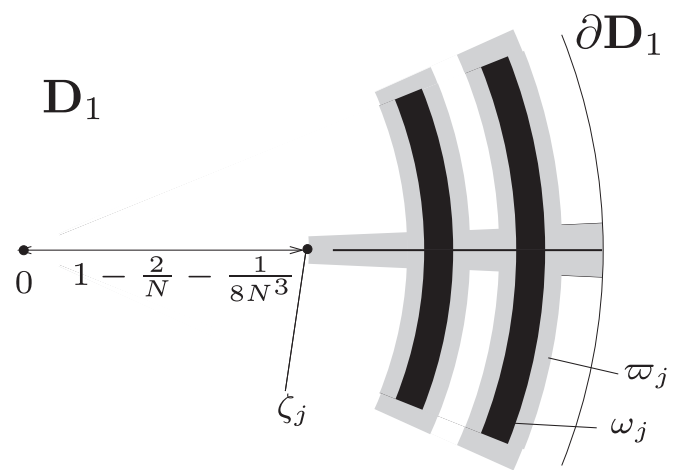

FIGURE 3. The base point $\zeta_{j}$ 
By definition, we have

Lemma A.1. (1) For each $j=1, \ldots, 2 N$, both $\omega_{j}$ and $\overline{\mathbf{D}}_{1} \backslash \varpi_{j}$ are disjoint compact subsets of $\mathbf{C}$ such that $\mathbf{C} \backslash\left(\omega_{j} \cup\left(\overline{\mathbf{D}}_{1} \backslash \varpi_{j}\right)\right)$ is connected.

(2) It holds that

$$
\overline{\mathbf{D}}_{1} \backslash \mathbf{D}_{1-\frac{2}{N}-\frac{1}{8 N^{3}}} \supset \varpi_{1} \cup \cdots \cup \varpi_{2 N} .
$$

Lemma A.2. Let $j \in\{1, \ldots, 2 N\}$. Then for each $p \in \overline{\mathbf{D}}_{1} \backslash \varpi_{j}$, there exists a path $\gamma$ in $\overline{\mathbf{D}}_{1} \backslash \varpi_{j}$ joining 0 and $p$ whose length (with respect to the Euclidean metric of $\mathbf{C )}$ is not greater than $1+\pi / N$.

Proof. By a rotation and a reflection on $\mathbf{C}=\mathbf{R}^{2}$, we assume $j=2 N$ and $p=r e^{\mathrm{i} \theta}(0 \leq r \leq 1,0 \leq \theta \leq \pi)$ without loss of generality.

If $\pi / N<\theta<\pi$, the line segment $\gamma$ joining 0 and $p$ does not intersect with $\varpi_{2 N}$. Then $\gamma$ is the desired path.

Otherwise, both the line segment $\gamma_{1}$ joining 0 and $p_{0}:=r e^{\mathrm{i} \pi / N}$ and the circular arc $\gamma_{2}$ joining $p_{0}$ and $p$ centered at 0 do not intersect with $\varpi_{2 N}$. Then the path $\gamma:=\gamma_{1} \cup \gamma_{2}$ is the desired one.

Lemma A.3. Let $j \in\{1, \ldots, 2 N\}$. Then for each $p \in \bar{\varpi}_{j}$, there exists a path $\gamma$ in $\bar{\varpi}_{j}$ joining the base point $\zeta_{j}$ and $p$ whose length (with respect to the Euclidean metric of $\mathbf{C}$ ) is not greater than $6 / N$.

Proof. We write $p=r e^{\mathrm{i} \theta} \in \bar{\varpi}_{j}$, where

$$
1-\frac{2}{N}-\frac{1}{8 N^{3}} \leq r \leq 1, \quad \frac{\pi(j-1)}{N} \leq \theta \leq \frac{\pi(j+1)}{N} .
$$

Then the line segment $\gamma_{1}$ joining $\zeta_{j}$ and $p_{1}:=r e^{\mathrm{i} \pi j / N}$ lies in $\bar{\varpi}_{j}$, and its Euclidean length does not exceed $\frac{2}{N}+\frac{1}{8 N^{3}}$. On the other hand, the length of the circular arc $\gamma_{2}$ centered at the origin joining $p_{1}$ and $p$ does not exceed $\pi / N$. Then the path $\gamma=\gamma_{1} \cup \gamma_{2}$ joins $\zeta_{j}$ and $p$ in $\bar{\varpi}_{j}$, whose length does not exceed

$$
\frac{2}{N}+\frac{1}{8 N^{3}}+\frac{\pi}{N}=\frac{1}{N}\left(2+\frac{1}{8 N^{2}}+\pi\right) \leq \frac{1}{N}\left(2+\frac{1}{8}+\pi\right) \leq \frac{6}{N} .
$$

Hence we have the conclusion. that

Lemma A.4. Assume $N \geq 4$, and let $\Omega \subset \mathbf{D}_{1}$ be the set as in (A.7). Note

$$
\Omega \subset \omega_{1} \cup \cdots \cup \omega_{2 N} .
$$


Consider a Riemannian metric $d s^{2}=\lambda^{2}|d z|^{2}$ on $\overline{\mathbf{D}}_{1}$ such that

$$
\begin{cases}\lambda \geq 1 & \left(\text { on } \overline{\mathbf{D}}_{1}\right) \\ \lambda \geq N^{3} & (\text { on } \Omega) .\end{cases}
$$

Then for an arbitrary path $\sigma$ in $\overline{\mathbf{D}}_{1}$ joining 0 and $\partial \mathbf{D}_{1}$, it holds that $\int_{\sigma} d s \geq N$.

Proof. For $k=0, \ldots, N^{2}-1$, let $\gamma_{k}$ be a subarc of $\sigma$ joining $\partial \mathbf{D}_{r_{2 k}}$ and $\partial \mathbf{D}_{r_{2 k+2}}$ contained in $\overline{\mathbf{D}}_{r_{2 k}} \backslash \mathbf{D}_{r_{2 k+2}}$. It suffices to prove that $\operatorname{Length}_{d s^{2}}\left(\gamma_{k}\right) \geq \frac{1}{N}$. In this case, since the path $\sigma$ contains at least $N^{2}$ such paths, we have

$$
\operatorname{Length}_{d s^{2}}(\sigma)=\int_{\sigma} d s \geq N^{2} \cdot \frac{1}{N}=N
$$

In order to prove that Length ${ }_{d s^{2}}\left(\gamma_{k}\right) \geq \frac{1}{N}$, we distinguish two cases. First we assume that Length ${ }_{\mathbf{C}}\left(\gamma_{k}\right) \geq \frac{1}{N}$. In this case by the assumption $\lambda \geq 1$ we have

$$
\operatorname{Length}_{d s^{2}}\left(\gamma_{k}\right)=\int_{\gamma_{k}} d s=\int_{\gamma_{k}} \lambda(z)|d z| \geq \int_{\gamma_{k}}|d z| \geq \frac{1}{N}
$$

On the contrary, if $\operatorname{Length}_{\mathbf{C}}\left(\gamma_{k}\right)<\frac{1}{N}$ it is not difficult to see that $\gamma_{k}$ must be contained in a sector of $\overline{\mathbf{D}}_{1}$ of angle bounded by $\frac{\pi}{N}-\frac{2}{N^{2}}$. Taking into account the shape of the labyrinth, this implies that $\gamma_{k}$ crosses a connected component of $\Omega$ transversely, and therefore the Euclidean length of $\gamma_{k} \cap \Omega$ is greater than $1 /\left(2 N^{3}\right)$. Hence by the assumption,

$$
\text { Length }_{d s^{2}}\left(\gamma_{k}\right)=\int_{\gamma_{k}} d s \geq \int_{\gamma_{k} \cap \Omega} d s=\int_{\gamma_{k} \cap \Omega} \lambda|d z| \geq N^{3} \cdot \frac{1}{2 N^{3}}=\frac{1}{2}>\frac{1}{N} .
$$

\section{REFERENCES}

[ 1 ] A. Alarcón And F. Forstnerič, Every bordered Riemann surface is a complete proper curve in a ball, Math. Ann. 377 (2013), 1049-1070.

[2] A. Alarcón and F. Forstnerič, Null curves and directed immersions of open Riemann surfaces, to appear in Invent. Math., arXiv:1210.5617v1.

[ 3 ] A. Alarcón and F. López, Null curves in $C^{3}$ and Calabi-Yau conjectures, Math. Ann. 355 (2013), 429-455.

[ 4 ] P. Collin and H. Rosenberg, Notes sur la démonstration de N. Nadirashvili des conjectures de Hadamard et Calabi-Yau, Bull. Sci. Math. 123 (1999), 563-576.

[5] F. Martín, M. Umehara and K. Yamada, Complete bounded null curves immersed in $\mathbf{C}^{3}$ and SL(2,C), Calculus of Variations and PDE's 36 (2009), 119-139.

Erratum: Complete boudded null curves immersed in $\mathbf{C}^{3}$ and $\operatorname{SL}(2, \mathbf{C})$, Calculus of Variations and PDE's 46 (2013), 439-440. 
[6] F. Martín, M. Umehara and K. Yamada, Complete bounded holomorphic curves immersed in $\mathbf{C}^{2}$ with arbitrary genus, Proc. Amer. Math. Soc. 137 (2009), 3437-3450.

[7] N. Nadirashvili, Hadamard's and Calabi-Yau's conjectures on negatively curved and minimal surfaces, Invent. Math. 126 (1996), 457-465.

\section{Leonor Ferrer}

Departamento de Geometría y Topología

UNIVERSIDAD DE GRANADA

18071 GRANADA

SPAIN

E-mail: 1ferrer@ugr.es

Francisco Martín

Departamento de Geometría y Topología

UNIVERSIDAD DE GRANADA

18071 GRANADA

SPAIN

E-mail: fmartin@ugr.es

Masaaki Umehara

Department of Mathematical and Computing Sciences

TOKYo Institute of TeChNOLOGY

2-12-1-W8-34, O-OKAYAMA

Meguro-Ku, TOKyo 152-8552

JAPAN

E-mail: umehara@is.titech.ac.jp

Kotaro Yamada

DePartment of Mathematics

Tokyo Institute of Technology

2-12-1-H-7, O-OKAYAMA

MEguro-KU, TOKYO 152-8551

JAPAN

E-mail: kotaro@math.titech.ac.jp 